Submitted to the Annals of Statistics

arXiv: arXiv: 1304.2085

\title{
SUPPLEMENTARY ARTICLE TO MINIMAX RISK OF MATRIX DENOISING BY SINGULAR VALUE THRESHOLDING
}

\author{
By David DONOHO ${ }^{*, \ddagger}$ AND Matan GaVish ${ }^{\dagger, *, \ddagger}$ \\ Stanford University $y^{\ddagger}$
}

\section{CONTENTS}

1 A sufficient condition for SV-monotonicity . . . . . . . . . . . 2

1.1 Proof of Lemma $2 \ldots \ldots \ldots$

2 Convexity of the Stein Unbiased Risk Estimate for SVST . . . . . 4

2.1 Proof of Lemma $3 \ldots \ldots \ldots$

3 Worst-case MSE . . . . . . . . . . . . . . . . . . 11

3.1 Proof of Lemma $5 \ldots \ldots \ldots$

4 Convexity of the minimax risk f . . . . . . . . . 19

4.1 Proof of Lemma $6 \ldots \ldots \ldots$

4.2 Proof of Lemma $9 \ldots \ldots \ldots$

5 Additional main results . . . . . . . . . . . . . . . . . 21

5.1 Proof of Theorem 5 . . . . . . . . . . . . . 21

5.2 Proof of Theorem $7 \ldots \ldots \ldots \ldots \ldots$

5.3 Proof of Theorem $6 \ldots \ldots \ldots \ldots \ldots$

5.4 Proof of Theorem 8. . . . . . . . . . . . . . 23

6 Singular-value denoisers can only improve in worst-case . . . . . . 24

6.1 Proof of Lemma $10 \ldots \ldots$. . . . . . . . . . . 24

7 Additional Discussion . . . . . . . . . . . . . . . 25

7.1 Another Connection between natrix SVST and sparse vector

estimation: Block Thresholding . . . . . . . . . . 25

7.2 Block Thresholding and the Minimaxity Gap . . . . . . . . 26

Acknowledgements . . . . . . . . . . . . . . . . . 27

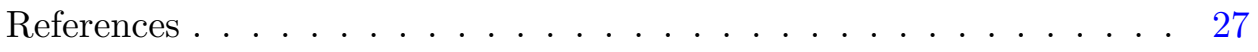

Author's addresses . . . . . . . . . . . . . . . . . . 29

\footnotetext{
*Partially supported by NSF DMS 0906812 (ARRA)

${ }^{\dagger}$ Partially supported by a William R. and Sara Hart Kimball Stanford Graduate Fellowship and a Technion EE Sohnis Promising Scientist Award
} 
1. A sufficient condition for SV-monotonicity. In this supplement section we prove Lemma 2, whereby a sum of bounded, orthogonally invariant, quasi-convex functions is $\mathrm{SV}$-monotone increasing.

Let us first show that in order to establish that a function $f$ is SVmonotone increasing, it is enough to show that $f$ is $\mathrm{SV}$-monotone increasing with respect to each singular value individually.

Lemma 11. Let $f: M_{m \times n} \rightarrow \mathbb{R}$ be an orthogonally invariant function such that

$$
\mathbb{E} f\left(\mathbf{a}_{\Delta}+Z\right) \leq \mathbb{E} f\left(\left(\mathbf{a}+\delta \mathbf{e}_{\mathbf{i}}\right)_{\Delta}+Z\right)
$$

for any $\mathbf{a} \in \mathbb{R}^{m}, \delta>0$ and $1 \leq i \leq m$, where $\mathbf{e}_{\mathbf{i}}$ is the canonical basis vector, $\left(\mathbf{e}_{\mathbf{i}}\right)_{j}=\delta_{i, j}$. Then $f$ is SV-monotone increasing.

Proof. Let $A, B \in M_{m \times n}$ with singular value decompositions $A=U_{A}$. $\mathbf{a}_{\Delta} \cdot V_{A}^{\prime}$ and $B=U_{B} \cdot \mathbf{b}_{\Delta} \cdot V_{B}^{\prime}$, and assume $A \preceq B$, namely $a_{i} \leq b_{i}, i=$ $1, \ldots, m$. Since $f$ is orthogonally invariant and $U_{A}^{\prime} \cdot Z \cdot V_{A} \sim Z$, we have $\mathbb{E} f(A+Z)=\mathbb{E} f\left(U_{A}^{\prime} \cdot A \cdot V_{A}+U_{A}^{\prime} \cdot Z \cdot V_{A}\right)=\mathbb{E} f\left(\mathbf{a}_{\Delta}+Z\right)$ and similarly $\mathbb{E} f(B+Z)=\mathbb{E} f\left(b_{\Delta}+Z\right)$. By assumption,

$$
\begin{aligned}
\mathbb{E} f(A+Z) & =\mathbb{E} f\left(\left(a_{1}, \ldots, a_{m}\right)_{\Delta}+Z\right) \\
& \leq \mathbb{E} f\left(\left(b_{1}, a_{2} \ldots, a_{m}\right)_{\Delta}+Z\right) \\
& \ldots \\
& \leq \mathbb{E} f\left(\left(b_{1}, \ldots, b_{m-1}, a_{m}\right)_{\Delta}+Z\right) \\
& \leq \mathbb{E} f\left(\left(b_{1}, b_{2} \ldots, b_{m-1}, b_{m}\right)_{\Delta}+Z\right)=\mathbb{E} f(B+Z) .
\end{aligned}
$$

Das Gupta, Anderson and Mudholkar [5, thm. 1] gave a useful extension of the seminal monotonicity result of Anderson [1]; we present it using our standing notation.

TheOREM 10. Let $W \in M_{m \times n}$ be a random matrix whose rows $\mathbf{w}_{j} \in \mathbb{R}^{n}$ $(1 \leq j \leq m)$ are mutually independent, each with a vector normal distribution $\mathbf{w}_{j} \sim \mathcal{N}\left(k_{j} \mathbf{x}_{j}, \Sigma_{j}\right)$, where $k_{j} \geq 0$ and $\mathbf{w}_{j} \in \mathbb{R}^{n}$. Assume that $E \subset M_{m \times n}$ is a convex set, symmetric in each $\mathbf{w}_{j}$ given the other $\mathbf{w}_{\ell}(\ell \neq j)$, in the sense that $W \in E$ iff $S \cdot W \in E$ for any $S \in M_{m \times m}$ with $S_{\Delta} \in\{ \pm 1\}^{m}$. Then $\mathbb{P}(W \in E)$ is monotone non-increasing in each, $k_{j}$ as long as $k_{j} \geq 0$. 
Theorem 10 asserts monotonicity of expectation of indicators. Using the co-area formula for expectation of non-negative random variables, we obtain monotonicity of expectation of quasi-concave (and hence quasi-convex) functions:

LEMMA 12. Bounded invariant quasi-convex functions are SVmonotone increasing. Let $f: M_{m \times n} \rightarrow \mathbb{R}$ be a bounded orthogonally invariant function. Assume that $f$ is quasi-convex function, in the sense that for all $c \in \mathbb{R}$ the set $f^{-1}((-\infty, c])$ is convex in $M_{m \times n}$. Then $f$ is SVmonotone increasing.

Proof. Let $\mathbf{a} \in \mathbb{R}^{m}, \delta>0$ and $1 \leq i \leq m$. Define $\mathbf{b}=\mathbf{a}+\delta \mathbf{e}_{\mathbf{i}}$. By Lemma 11, it is enough to show that

$$
\mathbb{E} f\left(\mathbf{a}_{\Delta}+Z\right) \leq \mathbb{E} f\left(\mathbf{b}_{\Delta}+Z\right) .
$$

Let $g=-f$ so that $g$ is quasi-concave, orthogonally invariant and bounded. Without loss of generality we may assume that inf $g=0$. By the co-area formula we have for the expectation of $g\left(\mathbf{a}_{\Delta}+Z\right)$ :

$$
\mathbb{E} g\left(\mathbf{a}_{\Delta}+Z\right)=\int_{0}^{\infty} \mathbb{P}\left\{g\left(\mathbf{a}_{\Delta}+Z\right) \geq c\right\} d c
$$

and it is therefore enough to show that for all $c \geq 0$,

$$
\mathbb{P}\left\{g\left(\mathbf{a}_{\Delta}+Z\right) \geq c\right\} \geq \mathbb{P}\left\{g\left(\mathbf{b}_{\Delta}+Z\right) \geq c\right\} .
$$

We invoke Anderson monotonicity, Theorem 10, with the value of $i$ chosen above, $k_{i}=a_{i}, \mathbf{w}_{i}$ the standard basis vector $\left(w_{i}\right)_{\ell}=\delta_{i, \ell}$ and $\Sigma_{i}=I_{n}$, and with $E=g^{-1}([c, \infty))$ above. Since $g$ is quasi-concave, $E$ is a convex set in $M_{m \times n}$. Since $f$ (and hence $g$ ) is orthogonally invariant $g\left(\mathbf{a}_{\Delta}+Z\right)=$ $g\left(S \cdot\left(\mathbf{a}_{\Delta}+Z\right)\right)$, namely $g$ is invariant under row sign changes, the symmetry requirement in the theorem is satisfied. The theorem therefore holds, and we obtain

$$
\mathbb{P}\left\{\mathbf{a}_{\Delta}+Z \in g^{-1}([c, \infty))\right\} \geq \mathbb{P}\left\{\mathbf{b}_{\Delta}+Z \in g^{-1}([c, \infty))\right\}
$$

as required.

1.1. Proof of Lemma 2. It is easy to verify that a sum of SV-monotone increasing function is itself $\mathrm{SV}$-monotone increasing. The result now follows from Lemma 12. 
2. Convexity of the Stein Unbiased Risk Estimate for SVST. In this supplement section we prove Lemma 3, whereby the Stein Unbiased Risk Estimate (SURE) for SVST is given as a finite sum of bounded, orthogonally invariant and quasi-convex functions.

We will need the following notation. Following [21, 20], we say that a function $f: \mathbb{R}^{m} \rightarrow \mathbb{R}$ is absolutely symmetric if it is invariant under permutations and sign changes of its coordinates. As discussed in Section 8, the function of a matrix argument $F: M_{m \times n} \rightarrow \mathbb{R}$ defined by $F(X)=f(\mathbf{x})$, where $X=$ $U_{X} \cdot \mathbf{x}_{\Delta} \cdot V_{X}^{\prime}$ for some orthogonal $U_{X} \in O_{m}$ and $V_{X} \in O_{n}$, is well defined only if $f$ is absolutely symmetric. Denote by $W_{m}=\left\{y_{1} \geq \cdots \geq y_{m} \geq 0\right\} \subset \mathbb{R}^{m}$ the set of singular value vectors (in non-increasing order) of matrices in $M_{m \times n}$ and by $W_{m}^{+}=\left\{y_{1}>\cdots>y_{m}>0\right\} \subset \mathbb{R}^{m}$ the space of non-degenerate singular value vectors. We denote by $\Sigma: M_{m \times n} \rightarrow W_{m}$ the map $Y \mapsto \mathbf{y}$ that maps a matrix $Y$ to its singular value vector $\mathbf{y}$, sorted in non-increasing order. $\Sigma$ is orthogonally invariant, namely invariant under the transformation $X \mapsto U \cdot X \cdot V^{\prime}$ for any orthogonal $U \in O_{m}$ and $V \in O_{n}$. Also note that if $Y=X+\sigma Z \in M_{m \times n}$ with $Z_{i, j} \stackrel{\text { iid }}{\sim} \mathcal{N}(0,1)$, then the event $\Sigma(Y) \in W_{m}^{+}$ holds almost surely.

Let $\hat{X}$ be a weakly differentiable estimator of $X_{0}$ from data $Y=X_{0}+\sigma Z$, where $Z$ has i.i.d standard normal entries. The Stein Unbiased Risk Estimate [31] is a function of the data, $Y \mapsto S U R E(Y)$, for which $\mathbb{E} S U R E(Y)=$ $\mathbb{E}\left\|\hat{X}-X_{0}\right\|_{2}^{2}$. In our case, $X_{0}, Z$ and $Y$ are matrices in $M_{m \times n}$, and Stein's theorem [31, thm. 1] implies that for

$$
\begin{aligned}
S U R E(Y) & =m n \sigma^{2}+\|\hat{X}(Y)-Y\|_{F}^{2} \\
& +2 \sigma^{2} \sum_{i, j} \frac{\partial(\hat{X}(Y)-Y)_{i, j}}{\partial Y_{i, j}}
\end{aligned}
$$

we have

$$
\left\|\hat{X}-X_{0}\right\|_{F}^{2}=\mathbb{E}_{X_{0}} S U R E(Y) .
$$

The following lemma specializes the general SURE formula (2.1) to the case of matrix estimators that act through the data singular values. This calculation has been carried out independently by [4]. While here we use the SURE formula for a theoretical purpose, [4] proposed to use it to select a threshold for matrix denoising by SVST in applications.

Lemma 13. Write the singular value decomposition of $Y \in M_{m \times n}$ as $Y=U_{Y} \cdot \mathbf{y}_{\Delta} \cdot V_{Y}^{\prime}$. Let $\hat{X}$ be any weakly differentiable element of $\mathcal{D}$, as in 
Definition 5. Consider $\hat{X}$ as an estimator of $X_{0}$ from $Y=X_{0}+\sigma Z$. Then for any $Y \in M_{m \times n}$ such that $\Sigma(Y) \in W_{m}^{+}$, we have $\operatorname{SURE}(Y)=\operatorname{sure}(\Sigma(Y))$, where sure $: W_{m}^{+} \rightarrow \mathbb{R}$ is given by

$$
\begin{aligned}
\operatorname{sure}(\mathbf{y}) & =m n \sigma^{2}+\|g(\mathbf{y})\|^{2}+2 \sigma^{2}\left[\sum_{1 \leq i \neq j \leq m} \frac{g(\mathbf{y})_{j} y_{j}-g(\mathbf{y})_{i} y_{i}}{y_{j}^{2}-y_{i}^{2}}\right. \\
& \left.+\left.\sum_{i=1}^{m} \frac{\partial g_{i}}{\partial y_{i}}\right|_{\mathbf{y}}+(n-m) \sum_{i=1}^{m} \frac{g(\mathbf{y})_{i}}{y_{i}}\right] .
\end{aligned}
$$

Here, $g(\mathbf{y})=\hat{\mathbf{x}}-\mathbf{y}$ and $\partial / \partial y_{i}$ is a weak derivative.

Note that $\mathbf{y} \mapsto \operatorname{sure}_{\lambda}(|\mathbf{y}|)$ is absolutely symmetric. Below, we extend the domain of sure $_{\lambda}$ by symmetry and consider sure $_{\lambda}: \mathbb{R}^{m} \rightarrow \mathbb{R}^{m}$.

Let us first show that Lemma 3, whereby $S U R E_{\lambda}$ is a bounded, orthogonally invariant and quasi-convex function of a matrix argument, follows from Lemma 13. To establish quasi-convexity if $S U R E_{\lambda}$ we will need to relate the quasi-convexity of $S U R E_{\lambda}$, a function of a matrix argument, to quasi-convexity of sure $_{\lambda}$, a function of singular values.

Lemma 14 . Let $f: \mathbb{R}^{m} \rightarrow \mathbb{R}$ be an absolutely symmetric and lowersemicontinuous function. If $f$ is a quasi-convex then the function $F: M_{m \times n} \rightarrow$ $\mathbb{R}$ defined by $F(X)=f(\Sigma(X))$ is quasi-convex on $M_{m \times n}$.

Proof. We use the following characterization of quasi-convexity by subgradients due to Aussel [2, thm. 2.2]: A lower semicontinuous function $g$ on a Banach space $\mathcal{X}$ is quasi-convex if and only if the following condition holds for all $\mathbf{x}, \mathbf{y} \in \mathcal{X}$ :

$$
\begin{gathered}
\exists \mathbf{x}^{*} \in \partial f(\mathbf{x}):\left\langle\mathbf{x}^{*}, \mathbf{y}-\mathbf{x}\right\rangle>0 \Longrightarrow \\
\forall \mathbf{y}^{*} \in \partial f(\mathbf{y}):\left\langle\mathbf{y}^{*}, \mathbf{y}-\mathbf{x}\right\rangle \geq 0 .
\end{gathered}
$$

Now, Lewis [20, cor. 2.5] has provided the following characterization of subgradients of $F$. Let $X, Y \in M_{m \times n}$. Then $Y \in \partial F(X)$ if and only if $\Sigma(Y) \in \partial f(\Sigma(X))$ and there exist orthogonal matrices $U \in O_{m}$ and $V \in O_{n}$ and vectors $\mathbf{x}, \mathbf{y} \in \mathbb{R}^{m}$ such that

$$
\begin{aligned}
& X=U \cdot \mathbf{x}_{\Delta} \cdot V \quad \text { and } \\
& Y=U \cdot \mathbf{y}_{\Delta} \cdot V .
\end{aligned}
$$

We also recall von Neumann's inequality for singular values [20, thm 2.1], whereby if $X, Y \in M_{m \times n}$ have singular value vectors $\mathbf{x}, \mathbf{y} \in \mathbb{R}^{m}$, then 
$\langle X, Y\rangle=\langle\mathbf{x}, \mathbf{y}\rangle$. Here and below, $\langle X, Y\rangle=\sum_{i, j} X_{i, j} Y_{i, j}$ is the Euclidean inner product on $M_{m \times n}$.

We finally turn to the proof. By [22, thm. 4.2], $F$ is lower-semicontinuous. Let us show that $F$ is quasi-convex using the characterization (2.3). Let $X, Y \in M_{m \times n}$ and assume that $X^{*} \in \partial F(X)$ exists such that $\left\langle X^{*}, Y-X\right\rangle>$ 0 . We now show that for all $Y^{*} \in \partial F(Y)$, we have $\left\langle Y^{*}, Y-X\right\rangle \geq 0$. Let $Y^{*} \in \partial F(Y)$, and let $\mathbf{x}, \mathbf{y}, \mathbf{x}^{*}$ and $\mathbf{y}^{*}$ denote the singular value vectors of $X, Y, X^{*}$ and $Y^{*}$ respectively. Since $X^{*} \in \partial F(X)$ we have $\left\langle X^{*}, X\right\rangle=\left\langle\mathbf{x}^{*}, \mathbf{x}\right\rangle$. Also, by von-Neumann's inequality we have $\left\langle X^{*}, Y\right\rangle \leq\left\langle\mathbf{x}^{*}, \mathbf{y}\right\rangle$. We therefore have

$$
\left\langle\mathbf{x}^{*}, \mathbf{x}\right\rangle=\left\langle X^{*}, X\right\rangle\left\langle\left\langle X^{*}, Y\right\rangle \leq\left\langle\mathbf{x}^{*}, \mathbf{y}\right\rangle\right.
$$

Since $f$ is quasi-convex, by assumption, in particular for $\mathbf{y}^{*}$ we have $\left\langle\mathbf{y}^{*}, \mathbf{x}\right\rangle \leq$ $\left\langle\mathbf{y}^{*}, \mathbf{y}\right\rangle$, since $\mathbf{y}^{*} \in \partial F(Y)$. Also, $\left\langle Y^{*}, Y\right\rangle=\left\langle\mathbf{y}^{*}, \mathbf{y}\right\rangle$. Again by von-Neumann's inequality we have $\left\langle Y^{*}, X\right\rangle \leq\left\langle\mathbf{y}^{*}, \mathbf{x}\right\rangle$. Together, this gives

$$
\left\langle Y^{*}, X\right\rangle \leq\left\langle\mathbf{y}^{*}, \mathbf{x}\right\rangle \leq\left\langle\mathbf{y}^{*}, \mathbf{y}\right\rangle=\left\langle Y^{*}, Y\right\rangle,
$$

as required.

Corollary 1. Suppose that $f: \mathbb{R}^{m} \rightarrow \mathbb{R}$ is representable as $f(\mathbf{y})=$ $\sum_{i=1}^{m} f_{1}\left(y_{i}\right)$ where $f_{1}: \mathbb{R} \rightarrow \mathbb{R}$ is a bounded, nondecreasing, lower-semicontinuous function such that $f_{1}(-y)=f_{1}(y)$. Then $F: M_{m \times n} \rightarrow \mathbb{R}$ defined by $F(X)=f(\Sigma(X))$ is bounded and quasi-convex.

Corollary 2. Suppose that $f: \mathbb{R}^{m} \rightarrow \mathbb{R}$ is representable as $f(\mathbf{y})=$ $\sum_{1 \leq i \neq j \leq m} f_{2}\left(y_{i}, y_{j}\right)$, where $f_{2}: \mathbb{R}^{2} \rightarrow \mathbb{R}$ is a quasi-convex, lower-semicontinuous function obeying $f_{2}(x, y)=f_{2}( \pm y, \pm x)$. Then $F: M_{m \times n} \rightarrow \mathbb{R}$ defined by $F(X)=f(\Sigma(X))$ is quasi-convex.

2.1. Proof of Lemma 3. Write $S U R E_{\lambda}$ for the SURE corresponding to the SVST estimator $\hat{X}_{\lambda}$. By Lemma 13, let sure $_{\lambda}$ be the function of the singular values $S U R E_{\lambda}(Y)=\operatorname{sure}_{\lambda}(\Sigma(Y))$. Substituting $\hat{x}(\mathbf{y})_{i}=\left(y_{i}-\lambda\right)_{+}$ and $\sigma=1 / \sqrt{n}$ in $(2.2)$, we get $g(\mathbf{y})_{i}=-\min \left\{y_{i}, \lambda\right\}$, hence

$$
\begin{aligned}
\text { sure }_{\lambda}(\mathbf{y}) & =m \\
& +\sum_{i=1}^{m}\left[\left(\min \left\{y_{i}, \lambda\right\}\right)^{2}-\mathbf{1}_{\left\{y_{i}<\lambda\right\}}-\frac{(n-m) \cdot \min \left\{y_{i}, \lambda\right\}}{y_{i}}\right] \\
& -\frac{2}{n} \sum_{1 \leq i \neq j \leq m} \frac{\min \left\{y_{j}, \lambda\right\} y_{j}-\min \left\{y_{i}, \lambda\right\} y_{j}}{y_{j}^{2}-y_{i}^{2}}
\end{aligned}
$$


Each of the terms $\min \left\{y_{i}, \lambda\right\},-\mathbf{1}_{y_{i}<\lambda}$ and $-\min \left\{y_{i}, \lambda\right\} / y_{i}$ is non-decreasing, bounded, lower-semicontinuous, and therefore, by Corollary 1, the function implicitly defined by the RHS of (2.4) is bounded and quasi-convex as required. We now turn to the function implicitly defined by the sum in (2.5). Each element in the sum is given by $(2 / n) \cdot f_{\lambda}\left(y_{j}, y_{i}\right)$ for some $1 \leq i \neq j \leq m$, where $f_{\lambda}:[0, \infty)^{2} \rightarrow \mathbb{R}$ is given by

$$
\begin{aligned}
f_{\lambda}(x, y) & =-\frac{\min \{y, \lambda\} y-\min \{x, \lambda\} x}{y^{2}-x^{2}} \\
& = \begin{cases}-1 & 0<x, y \leq \lambda \\
\frac{\lambda x-y^{2}}{y^{2}-x^{2}} & 0<y \leq \lambda, x \geq \lambda \\
\frac{-\lambda}{x+y} & x \geq \lambda, y \geq \lambda\end{cases}
\end{aligned}
$$

with $f_{\lambda}(x, y)=f_{\lambda}(y, x)$ and $f_{\lambda}(x, x)$ defined to yield a continuous function. The function $f_{\lambda}$ is shown in Figure 1 . We now show that $f_{\lambda}$ can be written as a sum of four bounded, lower-semicontinuous, quasi-convex functions. Decompose $f_{\lambda}$ as

$$
\begin{aligned}
f_{\lambda}(x, y) & =-\mathbf{1}_{[0, \lambda] \times[0, \lambda]}(x, y) \\
& +\frac{\lambda x-y^{2}}{y^{2}-x^{2}} \cdot \mathbf{1}_{(\lambda, \infty) \times(0, \lambda)}(x, y) \\
& +\frac{x^{2}-\lambda y}{y^{2}-x^{2}} \cdot \mathbf{1}_{(0, \lambda) \times(\lambda, \infty)}(x, y) \\
& -\frac{\lambda}{x+y} \cdot \mathbf{1}_{(\lambda, \infty) \times(\lambda, \infty)} .
\end{aligned}
$$

The image of each of these terms lies in $[-1,0]$, hence each is bounded. To see that the second (and hence the third) and the fourth terms are quasi-convex, note that for $0 \leq c \leq 1$,

$$
\begin{aligned}
& \left\{(x, y) \in[0, \infty)^{2} \mid \frac{\lambda x-y^{2}}{y^{2}-x^{2}} \cdot \mathbf{1}_{(\lambda, \infty) \times(0, \lambda)}(x, y) \leq-c\right\} \\
& =\left\{(x, y) \in(\lambda, \infty) \times(0, \lambda) \mid(1-c) y^{2} \leq-c x^{2}+\lambda x\right\}
\end{aligned}
$$

and

$$
\begin{array}{r}
\left\{(x, y) \in[0, \infty)^{2} \mid-\frac{\lambda}{x+y} \cdot \mathbf{1}_{(\lambda, \infty) \times(\lambda, \infty)}(x, y) \leq-c\right\} \\
=\left\{(x, y) \in(\lambda, \infty) \times(\lambda, \infty) \mid x+y \leq \frac{\lambda}{c}\right\}
\end{array}
$$


These are easily seen to be convex sets in $\mathbb{R}^{2}$. We conclude that the function $f_{\lambda}$ can be decomposed $f_{\lambda}=\sum_{s=1}^{4} f_{\lambda}^{(s)}$, where each $f_{\lambda}^{(s)}$ is quasi-convex. It follows that the sum (2.5) can be decomposed into four terms $T_{1}+\cdots+T_{4}$, each of which is a sum of quasi-convex functions of pairs of singular values, $T_{s}=\sum_{i \neq j} f_{\lambda}^{(s)}\left(y_{i}, y_{j}\right)$. By Corollary 2 , each term $T_{s}$, for $1 \leq s \leq 4$, is a bounded, quasi-convex function on matrix space: $T_{s}: M_{m \times n} \mapsto \mathbb{R}$. It follows that $S U R E_{\lambda}$ is a sum of five bounded, quasi-convex functions on matrix space, and the proof is complete.
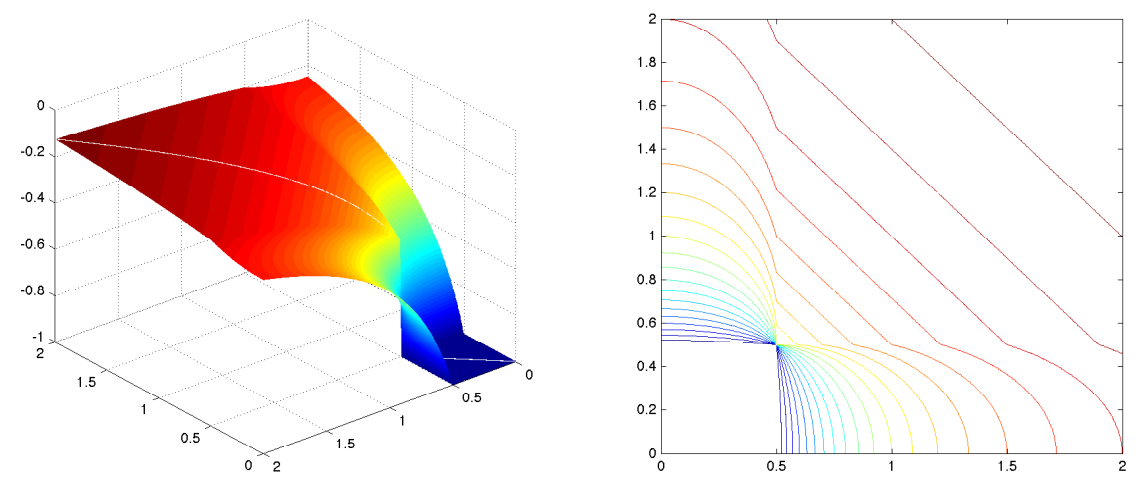

FIG 1. Part of the graph of the function $f_{\lambda}$ of (2.6). Left panel: rotated surface plot. Right panel: some level sets.

Proof of Lemma 13. We first calculate the Jacobian of the SVD, following [26]. For the reader's convenience, for the remainder of this section, consider the case $m \geq n$, in order to conform to their notation. We avoid differential geometry notation and restrict ourselves to the more cumbersome, but more widely used, multivariate calculus notation.

Let $Y=U_{Y} \cdot \mathbf{y}_{\Delta} \cdot V_{Y}^{\prime}$ denote a full ("fat") SVD of $Y \in M_{m \times n}$, where now $m \geq n$ and $\mathbf{y}_{\Delta} \in M_{m \times n}$. We can view $Y \mapsto U_{Y}$ as a map $M_{m \times n} \rightarrow M_{m \times m}$, where the first $n$ columns of $U_{Y}$ are determined (up to sign of each column) by $Y$ and the last $m-n$ columns constitute an arbitrary completion to an orthonormal basis of $\mathbb{R}^{m}$. Similarly $Y \mapsto y$ is a function into the Weyl chamber $\left\{\mathbf{y} \mid, y_{1} \geq \cdots \geq y_{n}\right\} \subset \mathbb{R}^{n}$ and $Y \mapsto V_{Y}$ is a map $M_{m \times n} \rightarrow M_{n \times n}$, determined up to sign of each column. As we will see later, the trace of the Jacobian of the SVD is well defined and does not depend on these arbitrary choices.

[26] have proposed to calculate the Jacobian of each of these multivariate 
real functions as follows. They show that

$$
\begin{aligned}
\frac{\partial U_{k, \ell}}{\partial Y_{i, j}} & =\left(U \cdot \Omega_{U}^{i, j}\right)_{k, \ell} \\
\frac{\partial V_{k, \ell}}{\partial Y_{i, j}} & =-\left(V \cdot \Omega_{V}^{i, j}\right)_{k, \ell} \\
\frac{\partial y_{k}}{\partial Y_{i, j}} & =U_{i, k} V_{j, k}
\end{aligned}
$$

for $1 \leq i, k \leq m$ and $1 \leq j, \ell \leq n$. Here, $\Omega_{U}^{i, j} \in M_{m \times n}$ and $\Omega_{V}^{i, j} \in M_{n \times n}$ for any $1 \leq i \leq m$ and $1 \leq j \leq n$. [26] show that both $\Omega_{V}^{i, j}$ and the upper $n \times n$ block of $\Omega_{U}^{i, j}$ are antisymmetric, and that each pair $\left(\left(\Omega_{U}^{i, j}\right)_{k, \ell},\left(\Omega_{V}^{i, j}\right)_{k, \ell}\right)$ with $1 \leq k, \ell \leq n$ satisfies the $2 \times 2$ linear system

$$
\begin{aligned}
& y_{\ell}\left(\Omega_{U}^{i, j}\right)_{k, \ell}+y_{k}\left(\Omega_{V}^{i, j}\right)_{k, \ell}=U_{i, k} V_{j, \ell} \\
& y_{k}\left(\Omega_{U}^{i, j}\right)_{k, \ell}+y_{\ell}\left(\Omega_{V}^{i, j}\right)_{k, \ell}=-U_{i, \ell} V_{j, k} .
\end{aligned}
$$

The authors do not explicitly provide the equations that determine the lower $(m-n) \times n$ block of $\Omega_{U}^{i, j}$. Fortunately, their arguments immediately imply that entries in this block satisfy

$$
y_{\ell}\left(\Omega_{U}^{i, j}\right)_{k, \ell}=U_{i, k} V_{j, \ell},
$$

for $1 \leq i \leq m, 1 \leq j, \ell \leq n$ and $n+1 \leq k \leq m$.

We can now evaluate the divergence term in (2.1),

$$
\sum_{i=1}^{m} \sum_{j=1}^{n} \frac{\partial(\hat{X}(Y)-Y)_{i, j}}{\partial Y_{i, j}}
$$

Define $G(Y)=\hat{X}-Y$ and $g(\mathbf{y})=\hat{\mathbf{x}}-\mathbf{y}$. To evaluate $\frac{\partial G(Y)_{i, j}}{\partial Y_{i, j}}$ we note that $G(Y)_{i, j}=\sum_{k} U_{i, k} g(\mathbf{y})_{k} V_{j, k}$ and hence

$$
\begin{aligned}
\frac{\partial G(Y)_{i, j}}{\partial Y_{i, j}} & =\sum_{k} \frac{\partial}{\partial Y_{i, j}}\left(U_{i, k} g(\mathbf{y})_{k} V_{j, k}\right)= \\
& =\sum_{k} \frac{\partial U_{i, k}}{\partial Y_{i, j}} g(\mathbf{y})_{k} V_{j, k}+ \\
& +\sum_{k} U_{i, k} g(\mathbf{y})_{k} \frac{\partial V_{j, k}}{\partial Y_{i, j}}+ \\
& +\sum_{k} U_{i, k} \frac{\partial g(\mathbf{y})_{k}}{\partial Y_{i, j}} V_{j, k} .
\end{aligned}
$$


Since

$$
\frac{\partial g(\mathbf{y})_{k}}{\partial Y_{i, j}}=\sum_{\ell=1}^{n} \frac{\partial g(\mathbf{y})_{k}}{\partial y_{\ell}} \frac{\partial y_{\ell}}{\partial Y_{i, j}}=\sum_{\ell=1}^{n} \frac{\partial g(\mathbf{y})_{k}}{\partial y_{\ell}} U_{i, \ell} V_{j, \ell}
$$

we conclude that

$$
\begin{aligned}
\frac{\partial G(Y)_{i, j}}{\partial Y_{i, j}} & =\sum_{k}\left(U \Omega_{U}^{i, j}\right)_{i, k} g(\mathbf{y})_{k} V_{j, k}+ \\
& -\sum_{k} U_{i, k} g(\mathbf{y})_{k}\left(V \Omega_{V}^{i, j}\right)_{j, k}+ \\
& +\sum_{k} U_{i, k} V_{j, k}\left(\sum_{\ell} \frac{\partial g(\mathbf{y})_{k}}{\partial y_{\ell}} U_{i, \ell} V_{i, \ell}\right) .
\end{aligned}
$$

Recall that the Jacobian trace $\operatorname{Tr}\left(\frac{\partial G}{\partial Y}\right)=\sum_{i, j} \frac{\partial G(Y)_{i, j}}{\partial Y_{i, j}}$ is invariant under change of basis of the underlying linear space $M_{m \times n}$. Consider the orthonormal basis of $M_{m \times n}$ given by rank-1 matrices, $\left\{\mathbf{u}_{i} \cdot \mathbf{v}_{j}^{\prime}\right\}_{i, j}$ where $\mathbf{u}_{1}, \ldots, \mathbf{u}_{m}$ are the columns of $U$ and $\mathbf{v}_{1}, \ldots, \mathbf{v}_{n}$ are the columns of $V$, respectively. To calculate the trace in this basis, we formally replace $U$ and $V$ with identity matrices of their respective dimensions.

The equations that determine $\Omega_{U}^{i, j}$ and $\Omega_{V}^{i, j}$ for $1 \leq i \leq m$ and $1 \leq j \leq n$, namely (2.10) and (2.11), become

$$
\begin{aligned}
& y_{\ell}\left(\Omega_{U}^{i, j}\right)_{k, \ell}+y_{k}\left(\Omega_{V}^{i, j}\right)_{k, \ell}=\delta_{i, k} \delta_{j, \ell} \\
& y_{k}\left(\Omega_{U}^{i, j}\right)_{k, \ell}+y_{\ell}\left(\Omega_{V}^{i, j}\right)_{k, \ell}=-\delta_{i, \ell} \delta_{j, k}
\end{aligned}
$$

for $1 \leq k, \ell \leq n$, and

$$
y_{\ell}\left(\Omega_{U}^{i, j}\right)_{k, \ell}=\delta_{i, k} \delta_{j, \ell},
$$

for $n+1 \leq k \leq m$ and $1 \leq \ell \leq n$. Similarly, in this basis, (2.12) becomes

$$
\begin{aligned}
\operatorname{Tr}\left(\frac{\partial G}{\partial Y}\right) & =\sum_{i=1}^{m} \sum_{j=1}^{n} \sum_{k=1}^{n}\left[\left(\Omega_{U}^{i, j}\right)_{i, k} g(\mathbf{y})_{k} \delta_{j, k}-\delta_{i, k} g(\mathbf{y})_{k}\left(\Omega_{V}^{i, j}\right)_{j, k}\right. \\
& \left.+\delta_{i, k} \delta_{j, k}\left(\sum_{\ell} \frac{\partial g(\mathbf{y})_{k}}{\partial y_{\ell}} \delta_{i, \ell} \delta_{i, \ell}\right)\right]= \\
& =\sum_{i=1}^{m} \sum_{\substack{j=1 \\
j \neq i}}^{n}\left[\left(\Omega_{U}^{i, j}\right)_{i, j} g(\mathbf{y})_{j}-g(\mathbf{y})_{i}\left(\Omega_{V}^{i, j}\right)_{j, i}\right]+\sum_{j=1}^{n} \frac{\partial g(\mathbf{y})_{j}}{\partial y_{j}}
\end{aligned}
$$


Solving (2.13) and (2.14) for $\left(\Omega_{U}^{i, j}\right)_{i, j}$ and $\left(\Omega_{V}^{i, j}\right)_{i, j}$, where $i \neq j$, we get

$$
\begin{aligned}
& \left(\Omega_{U}^{i, j}\right)_{i, j}=\frac{y_{j}}{y_{j}^{2}-y_{i}^{2}} \quad 1 \leq i, j \leq n \\
& \left(\Omega_{U}^{i, j}\right)_{i, j}=\frac{1}{y_{j}} \quad n+1 \leq i \leq m, 1 \leq j \leq n \\
& \left(\Omega_{V}^{i, j}\right)_{i, j}=-\frac{y_{i}}{y_{j}^{2}-y_{i}^{2}} \quad 1 \leq i, j \leq n
\end{aligned}
$$

and $\left(\Omega_{U}^{i, i}\right)_{i, i}=\left(\Omega_{V}^{i, i}\right)_{i, i}=0$, to the effect that

$$
\begin{aligned}
\operatorname{Tr}\left(\frac{\partial G}{\partial Y}\right) & =\sum_{1 \leq i \neq j \leq n} \frac{y_{j} g(\mathbf{y})_{j}-y_{i} g(\mathbf{y})_{i}}{y_{j}^{2}-y_{i}^{2}} \\
& +(m-n) \sum_{j=1}^{n} \frac{g(\mathbf{y})_{j}}{y_{j}}+\sum_{i=j}^{n} \frac{\partial g(\mathbf{y})_{j}}{\partial y_{j}}
\end{aligned}
$$

Changing back to our original notation of $m \leq n$ by exchanging the sumbols $m$ and $n$, and using the general SURE formula (2.1), we have proved Lemma 13.

3. Worst-case MSE. In this supplemental section we prove Lemma 5, which gives a formula for the finite- $n$ worst-case MSE. Lemma 5 depends on the following three lemmas, which contain crucial observations regarding the singular values of $X_{0}+Z$ in the case where $X_{0}$ is a rank $r$ matrix and is rescaled so $\left\|X_{0}\right\| \rightarrow \infty$.

Lemma 15. Let $Y_{\mu}=\mu X_{0}+Z=U_{\mu} \cdot\left(\mathbf{y}_{\mu}\right)_{\Delta} \cdot V_{\mu}^{\prime}$, where $X_{0} \in M_{m \times n}$ is of rank $r$ and and write $\mathbf{y}_{\mu}=\left(y_{\mu, 1}, \ldots, y_{\mu, m}\right)$ with $y_{\mu, 1} \geq \ldots \geq y_{\mu, m}$. Then

$$
\lim _{\mu \rightarrow \infty} y_{\mu, i}=\infty, \quad i=1, \ldots, r
$$

Lemma 16. Let $Y_{\mu}=\mu X_{0}+Z=U_{\mu} \cdot\left(\mathbf{y}_{\mu}\right)_{\Delta} \cdot V_{\mu}^{\prime}$, where $X_{0} \in M_{m \times n}$ is of rank $r$, and write $\mathbf{y}_{\mu}=\left(y_{\mu, 1}, \ldots, y_{\mu, m}\right)$ with $y_{\mu, 1} \geq \ldots \geq y_{\mu, m}$. Also, define

$$
\begin{aligned}
\mathbf{z}_{\mu} & =\left(U_{\mu}^{\prime} \cdot Z \cdot V_{\mu}\right)_{\Delta} \\
\mathbf{x}_{\mu} & =\mu \cdot\left(U_{\mu}^{\prime} \cdot X_{0} \cdot V_{\mu}\right)_{\Delta}
\end{aligned}
$$

with $\mathbf{z}_{\mu}=\left(z_{\mu, 1}, \ldots, z_{\mu, m}\right)$. Finally, let $\left(\zeta_{1}, \ldots, \zeta_{m-r}\right)=\zeta\left(X_{0}, Z \mid \mathbf{X}\right)$ as in Definition 4 , where $\mathbf{X}=$ Mat or $\mathbf{X}=$ Sym. Then, as $\mu \rightarrow \infty$, the sum 
$\sum_{i=1}^{r} z_{\mu, i}$ converges almost surely to a zero-mean Gaussian random variable. Furthermore, we have, almost surely,

$$
\begin{aligned}
\lim _{\mu \rightarrow \infty} y_{\mu, r+i} & =\left|\zeta_{i}\right| \\
\lim _{\mu \rightarrow \infty} z_{\mu, r+i} & =\zeta_{i} \\
\lim _{\mu \rightarrow \infty} x_{\mu, r+i} & =0
\end{aligned}
$$

for any $1 \leq i \leq m-r$.

Proof of Lemma 15. For a symmetric matrix $S$, let $\lambda_{i}(S)$ denote the $i$-th eigenvalue in nonincreasing order. We write $A \triangleleft B$ if $A$ is a linear subspace of $B$. The Courant-Fischer min-max characterization of eigenvalues [15] states that

$$
\lambda_{i}(S)=\min _{\substack{U \triangleleft \mathbb{R}^{m} \\ \operatorname{codim}(U)=i-1}} \max _{\substack{\mathbf{v} \in U \\\|\mathbf{v}\|=1}} \mathbf{v}^{\prime} S \mathbf{v}
$$

Note that the squared singular values of $Y_{\mu}$ obey $y_{i}^{2}=\lambda_{i}\left(Y_{\mu} Y_{\mu}^{\prime}\right)$.

Choose $1 \leq i \leq r$. Let $U \triangleleft \mathbb{R}^{m}$ with $\operatorname{codim}(U)=i-1$. Since $m-r+1 \leq$ $\operatorname{dim}(U) \leq m$ necessarily $U \cap \operatorname{Im}\left(X_{0}\right) \neq \emptyset$ and we can choose $\mathbf{v} \in U \cap \operatorname{Im}\left(X_{0}\right)$ with $\|\mathbf{v}\|=1$. For this $\mathbf{v}$, and with the $y_{i}$ again denoting the singular values of $Y_{\mu}$,

$$
y_{i}^{2} \geq \mathbf{v}^{\prime} Y_{\mu} Y_{\mu}^{\prime} \mathbf{v}, \quad 1 \leq i \leq r .
$$

Without loss of generality, we may assume we are working in the basis of the SVD of $X_{0}$, so that $X_{0}$ is vanishing except in the first $r$ positions of the diagonal. We let $G_{r}$ denote the $r \times r$ sub-matrix of the upper left corner of $X_{0} X_{0}^{\prime}$; this is the 'principal piece' of $X_{0} X_{0}^{\prime}$ where the non zeros live; the rest of $X_{0} X_{0}^{\prime}$ is vanishing in this basis. Also let $Z_{r}$ denote the upper left $r$ by $r$ corner of $Z$, and let $X_{r}$ denote the $r$ by $r$ diagonal matrix whose diagonal entries are the ordered singular values. Let $H$ denote the $m \times m$ matrix which is zero except in the upper left corner, where there is a nonzero $r \times r$ sub matrix $H_{r}$ given by $H_{r}=X_{r} Z_{r}+Z_{r}^{\prime} X_{r}^{\prime}$. We also need $W_{r}$, the upper left $r$ by $r$ corner of the Wishart matrix $Z Z^{\prime}$. Finally we note that when represented in this basis, $\mathbf{v}=\left(v_{r}, 0_{m-r}\right)$, i.e. the only non zeros in $\mathbf{v}$ occur in the first $r$ places, and we let $v_{r}$ denote the $r \times 1$ column vector of those first $r$ entries.

For a symmetric matrix $S$, let $\lambda_{\min }(S)$ denote the smallest eigenvalue. Note that $\lambda_{\min }\left(Z Z^{\prime}\right) \geq 0$; that $\lambda_{\min }\left(G_{r}\right)>0$ by our hypothesis that the rank of $X_{0}$ is $r$, and finally note that $\lambda_{\min }\left(H_{r}\right)$ may be positive or negative, but is in any case well-defined ${ }^{1}$.

\footnotetext{
${ }^{1}$ Moreover, for a fixed choice of $X_{0}$, one could control the distribution of $\lambda_{\min }\left(H_{r}\right)$
} 
Now $Y_{\mu} Y_{\mu}^{\prime}=\mu^{2}\left(X_{0} X_{0}^{\prime}\right)+\mu X_{0} Z^{\prime}+\mu Z X_{0}^{\prime}+Z Z^{\prime}$. Taking into account our choice of coordinates, the upper left $r$ by $r$ block of $Y_{\mu} Y_{\mu}^{\prime}$ is $\mu^{2} G_{r}+\mu H_{r}+W_{r}$. The vector $\mathbf{v}$ 'sees' only the first $r$ coordinates and

$$
\begin{aligned}
\mathbf{v}^{\prime} Y_{\mu} Y_{\mu}^{\prime} \mathbf{v} & =\mu^{2} v_{r}^{\prime} G_{r} v_{r}+\mu v_{r}^{\prime} H_{r} v_{r}+v_{r}^{\prime} W_{r} v_{r} \\
& \geq \mu^{2} \lambda_{\min }\left(G_{r}\right)+\mu \lambda_{\min }\left(H_{r}\right)+\lambda_{\min }\left(W_{r}\right)
\end{aligned}
$$

This lower bound does not depend on the specific choice of $U$ or of $\mathbf{v}$. Because $\lambda_{\min }\left(G_{r}\right)>0$, this lower bound tends to $\infty$ as $\mu \rightarrow \infty$; we finally invoke (3.5).

Proof of Lemma 16. Write $\bar{Y}_{\sigma}=\sigma Y(1 / \sigma)=X_{0}+\sigma Z$ and let $\bar{Y}_{\sigma}=$ $\bar{U}_{\sigma} \cdot\left(\mathbf{y}_{\sigma}\right)_{\Delta} \cdot \bar{V}_{\sigma}^{\prime}$ denote the SVD of $\bar{Y}$. Note that $U_{1 / \sigma}=\bar{U}_{\sigma}$ and $V_{1 / \sigma}=\bar{V}_{\sigma}$. Write

$$
\bar{U}_{\sigma}=\left(\begin{array}{ccc}
\mid & & \mid \\
\overline{\mathbf{u}}_{\sigma}^{1} & \cdots & \overline{\mathbf{u}}_{\sigma}^{m} \\
\mid & & \mid
\end{array}\right) \quad \bar{V}_{\sigma}=\left(\begin{array}{ccc}
\mid & & \mid \\
\overline{\mathbf{v}}_{\sigma}^{1} & \cdots & \overline{\mathbf{v}}_{\sigma}^{n} \\
\mid & & \mid
\end{array}\right),
$$

with $\overline{\mathbf{u}}_{\sigma}^{i} \in \mathbb{R}^{m}$ and $\overline{\mathbf{v}}_{\sigma}^{i} \in \mathbb{R}^{n}$, for the columns of $\bar{U}_{\sigma}$ and $\bar{V}_{\sigma}$. Similarly write $\mathbf{u}_{\mu}^{i}\left(\right.$ resp. $\left.\mathbf{v}_{\mu}^{i}\right)$ for the columns of $U_{\mu}$ (resp. $V_{\mu}$ ).

We start with the first part of the lemma, regarding $\sum_{i=1}^{r} z_{\mu, i}$. Assume first that the image of $X_{0}$ is non degenerate, namely, that $X_{0}$ has $r$ distinct nonzero singular values. Fix some $1 \leq i \leq r$. Because the $i$-th singular values of $X_{0}$ separated from 0 and from all the other singular values, we can apply the singular vector perturbation analysis in Theorem 8.6.5 of Golub and Van Loan [15] (originally based on P. Stewart's work) to obtain a representation of $\overline{\mathbf{u}}_{\sigma}^{i}$ and $\overline{\mathbf{v}}_{\sigma}^{i}$ and in terms of the $i$-th left and right singular vectors of $X_{0}$, namely $\overline{\mathbf{u}}_{0}^{i}$ and $\overline{\mathbf{v}}_{0}^{i}$. Specifically, by that theorem, there exist constants $C, \sigma_{0}$, and vectors $\mathbf{a}_{\sigma}, \mathbf{b}_{\sigma}$, such that $\left\|\mathbf{a}_{\sigma}\right\| \leq C \sigma,\left\|\mathbf{b}_{\sigma}\right\| \leq C \sigma$, and

$$
\overline{\mathbf{u}}_{\sigma}^{i}=\overline{\mathbf{u}}_{0}^{i}+\mathbf{a}_{\sigma} \quad \overline{\mathbf{v}}_{\sigma}^{i}=\overline{\mathbf{v}}_{0}^{i}+\mathbf{b}_{\sigma}
$$

for all $\sigma<\sigma_{0}$. It follows that $\lim _{\mu \rightarrow \infty} \mathbf{z}_{\mu, i}=\lim _{\sigma \rightarrow 0}\left(\overline{\mathbf{u}}_{\sigma}^{i}\right)^{\prime} Z \overline{\mathbf{v}}_{\sigma}^{i}=\left(\overline{\mathbf{u}}_{0}^{i}\right)^{\prime} Z \overline{\mathbf{v}}_{0}^{i}$. Since $\mathbf{u}_{0}^{i}$ and $\mathbf{v}_{0}^{i}$ are non-random vectors, the right hand side is a zero-mean Gaussian variable as required.

The degenerate case, where some singular values of $X_{0}$ have multiplicities, is more delicate as the perturbation $\sigma Z$ above removes the degeneracy, and consequently the limiting singular vectors associated with degenerate singular subspaces depend on $Z$. To keep the proof as simple as possible, we focus on the fully degenerate case, where $X_{0}$ has $r$ nonzero singular values all 
equal to $x>0$. Observe first that $\mathbf{z}_{\mu}$ is invariant under the map $Y \mapsto A^{\prime} Y B$ for any orthogonal matrices $A$ and $B$. Taking $A$ and $B$ to be the left and right singular vectors of $X_{0}$, we may assume that $X_{0}$ is diagonal. In block notation, we have

$$
\begin{aligned}
X_{0} & =\left(\begin{array}{c|c}
x I_{r \times r} & 0_{r \times(m-r)} \\
\hline 0_{(m-r) \times r} & 0_{(m-r) \times(n-r)}
\end{array}\right) \text { and } \\
Z & =\left(\begin{array}{c|c}
Z_{11} & Z_{12} \\
\hline Z_{21} & Z_{22}
\end{array}\right),
\end{aligned}
$$

where $I_{r \times r}$ is the $r$-by- $r$ identity matrix, $0_{k \times \ell}$ is the $k$-by- $\ell$ zero matrix, $Z_{11} \in M_{r \times r}$ and $Z_{22} \in M_{(m-r) \times(n-r)}$. Expanding $\bar{Y}_{\sigma}$ in this block notation, we find that

$$
\bar{Y}_{\sigma} \bar{Y}_{\sigma}^{\prime}=\left(\begin{array}{c|c}
x^{2} I_{r \times r}+x \sigma\left(Z_{11}+Z_{11}^{\prime}\right) & 0_{r \times r} \\
\hline 0_{r \times r} & 0_{r \times r}
\end{array}\right)+x \sigma M_{\sigma}
$$

where $M_{\sigma}$ is an $m$-by- $m$ symmetric matrix (that depends on $Z$ ) whose top eigenvalue is bounded in $\sigma$. For a fixed value of $Z$ and small enough $\sigma$, the eigenvalues of $x^{2} I_{r \times r}+x \sigma\left(Z_{11}+Z_{11}^{\prime}\right)$ are all nonzero, and we can apply the symmetric matrix perturbation Theorem 8.1.10 of Golub and Van Loan [15] (again based on P. Stewart's work), whereby there exists a constant $C$, which depends on $Z$, and an $(m-r)$-by- $r$ matrix $P$ with $\|P\|_{2} \leq C \sigma$ such that the columns of

$$
\left(\left(\frac{W}{0_{(m-r) \times r}}\right)+\left(\frac{0_{r \times(m-r)}}{I_{(m-r) \times(m-r)}}\right) P\right)\left(I+P^{\prime} P\right)^{1 / 2}
$$

are the top $r$ eigenvectors of $\bar{Y}_{\sigma} \bar{Y}_{\sigma}^{\prime}$, where here and below, $W \in M_{r \times r}$ is the orthogonal matrix diagonalizing $Z_{11}+Z_{11}^{\prime}$. It follows that

$$
\lim _{\sigma \rightarrow 0} \bar{U}_{\sigma}=\left(\begin{array}{c|c}
W & * \\
\hline 0_{(m-r) \times r} & *
\end{array}\right) .
$$

An identical argument shows that the top $r$ eigenvectors of $\bar{Y}_{\sigma}^{\prime} \bar{Y}_{\sigma}$ have the same limit, namely

$$
\lim _{\sigma \rightarrow 0} \bar{V}_{\sigma}=\left(\begin{array}{c|c}
W & * \\
\hline 0_{(n-r) \times r} & *
\end{array}\right) .
$$

As a result, the top $r$-by- $r$ minor of $\bar{U}_{\sigma}^{\prime} Z \bar{V}_{\sigma}$ is just $W^{\prime} Z_{11} W$ and we obtain

$$
\begin{aligned}
\lim _{\mu \rightarrow \infty} \sum_{i=1}^{r} z_{\mu, i} & =\lim _{\sigma \rightarrow 0} \sum_{i=1}^{r}\left(\bar{U}_{\sigma}^{\prime} Z \bar{V}_{\sigma}\right)_{i, i} \\
& =\operatorname{Tr}\left(W^{\prime} Z_{11} W\right)=\operatorname{Tr}\left(Z_{11}\right),
\end{aligned}
$$


a zero-mean Gaussian variable as required.

We now turn to the second part of the lemma, regarding $y_{\mu, i}, x_{\mu, i}$ and $z_{\mu, i}$ for $r+1 \leq i \leq m$. Let $\bar{U}_{\sigma, m-r}$ and $\bar{V}_{\sigma, n-r}$ denote matrices consisting of the last $m-r$ and $n-r$ columns of $\bar{U}_{\sigma}$ and $\bar{V}_{\sigma}$, again in the ordering where the largest singular value has index $i=1$. Similarly, let $\bar{U}_{0, r}$ and $\bar{V}_{0, r}$ denote the sub matrices of the first $r$ columns of $\bar{U}_{0}$ and $\bar{V}_{0}$.

As the $r$ nonzero singular values of $X_{0}$ are separated from 0 (regardless of their configuration), we can again appeal to the perturbation Theorem 8.6.5 of Golub and Van Loan [15] to obtain the representation

$$
\begin{aligned}
\bar{U}_{\sigma, m-r} & =\left(\bar{U}_{0, m-r}+\bar{U}_{0, r} P_{\sigma}\right) Q_{\sigma}, \\
\bar{V}_{\sigma, n-r} & =\left(\bar{V}_{0, n-r}+\bar{V}_{0, r} R_{\sigma}\right) S_{\sigma},
\end{aligned}
$$

where $P_{\sigma}, Q_{\sigma}, R_{\sigma}, S_{\sigma}$ all depend on $\sigma, X_{0}$, and $Z, P_{\sigma} \in M_{r \times m-r}, Q_{\sigma} \in$ $M_{m-r \times m-r}, R_{\sigma} \in M_{r \times n-r}, S_{\sigma} \in M_{n-r \times n-r}$. In short, the perturbed singular vectors are representable using the unperturbed ones. In this representation, the cited theorem provides the following control of the coefficients:

$$
\left\|P_{\sigma}\right\|_{2}=O(\sigma),\left\|R_{\sigma}\right\|_{2}=O(\sigma), \sigma \rightarrow 0 ; \quad\left\|Q_{\sigma}\right\|_{2} \leq 2,\left\|S_{\sigma}\right\|_{2} \leq 2, \sigma<\sigma_{0},
$$

where $\|\cdot\|_{2}$ is the operator norm on $\ell_{2}$. Remarking that

$$
\bar{U}_{0, m-r}^{\prime} X_{0}=0, \text { and } X_{0} \bar{V}_{0, n-r}=0,
$$

we have

$$
\bar{U}_{\sigma, m-r}^{\prime} X_{0} \bar{V}_{\sigma, n-r}=P_{\sigma}^{\prime} Q_{\sigma}^{\prime} \bar{U}_{0, r}^{\prime} X_{0} \bar{V}_{0, r} R_{\sigma} S_{\sigma}
$$

and so

$$
\begin{aligned}
\left|\left(\bar{U}_{\sigma, m-r}^{\prime} X_{0} \bar{V}_{\sigma, n-r}\right)_{i, i}\right| & \leq\left\|P_{\sigma}^{\prime} Q_{\sigma}^{\prime} \bar{U}_{0, r}^{\prime} X_{0} \bar{V}_{0, r} R_{\sigma} S_{\sigma}\right\|_{2} \\
& \leq\left\|P_{\sigma}^{\prime}\right\|_{2} \cdot\left\|Q_{\sigma}^{\prime}\right\|_{2} \cdot\left\|X_{0}\right\|_{2} \cdot\left\|R_{\sigma}\right\|_{2} \cdot\left\|S_{\sigma}\right\|_{2} \\
& =O\left(\sigma^{2}\right), \quad \sigma \rightarrow 0 .
\end{aligned}
$$

Recalling $\sigma=1 / \mu$, and $\mathbf{u}_{\mu}^{r+i}$ is the $i$-th column of $\bar{U}_{\sigma, m-r}$ with $\sigma=1 / \mu$, analogously for $\mathbf{v}_{\mu}^{r+i}$,

$$
\begin{aligned}
\lim _{\mu \rightarrow \infty} x_{\mu, r+i} & =\lim _{\mu \rightarrow \infty}\left(\mathbf{u}_{\mu}^{r+i}\right)^{\prime} \cdot\left(\mu X_{0}\right) \cdot \mathbf{v}_{\mu}^{r+i} \\
& =\lim _{\mu \rightarrow \infty} \mu \cdot\left|\left(\bar{U}_{1 / \mu, m-r}^{\prime} X_{0} \bar{V}_{1 / \mu, n-r}\right)_{i, i}\right| \\
& =\lim _{\mu \rightarrow \infty} \mu \cdot O\left(\mu^{-2}\right)=0
\end{aligned}
$$

for $1 \leq i \leq m-r$. 
The projectors $\Pi_{m}$ and $\Pi_{n}$ of Definition 4 obey $\overline{\mathbf{u}}_{0}^{r+i}=\Pi_{m} \overline{\mathbf{u}}_{0}^{r+i}$ and $\overline{\mathbf{v}}_{0}^{r+i}=\Pi_{n} \overline{\mathbf{v}}_{0}^{r+i}$ for $i=1, \ldots m-r$. As $\lim _{\sigma \rightarrow 0} \overline{\mathbf{u}}_{\sigma}^{i}=\lim _{\sigma \rightarrow 0} \Pi_{m} \overline{\mathbf{u}}_{\sigma}^{i}$ and $\lim _{\sigma \rightarrow 0} \overline{\mathbf{v}}_{\sigma}^{i}=\lim _{\sigma \rightarrow 0} \Pi_{n} \overline{\mathbf{v}}_{\sigma}^{i}$, it follows that for $1 \leq i \leq m-r$ we have $\lim _{\mu \rightarrow \infty} y_{\mu, r+i}=\left\|\Pi_{m}^{\prime} Y_{\mu} \Pi_{n}-A_{i}\right\|_{2}$, where $\|\cdot\|_{2}$ is the operator norm on $\ell_{2}$ and $A_{i} \in M_{(m-r) \times(n-r)}$ is the best rank- $i$ approximation of $\Pi_{m}^{\prime} Y_{\mu} \Pi_{n}=$ $\Pi_{m}^{\prime} Z \Pi_{n}$ in Frobenius norm, namely, $\lim _{\mu \rightarrow \infty} y_{\mu, r+i}=\zeta_{i}$. Finally, this implies $\lim _{\mu \rightarrow \infty} z_{\mu, r+i}=\lim _{\mu \rightarrow \infty} y_{\mu, r+i}=\lim _{\mu \rightarrow \infty} x_{\mu, r+i}=\zeta_{i}$.

3.1. Proof of Lemma 5. Assume for simplicity that the vector $\mathbf{y}$ of singular values of the data matrix is sorted in non-increasing order: $y_{1} \geq \ldots \geq y_{m}$. Define

$$
Y_{\mu}=\mu X_{0}+\frac{1}{\sqrt{n}} Z=U_{\mu} \cdot\left(\mathbf{y}_{\mu}\right)_{\Delta} \cdot V_{\mu}^{\prime}
$$

and write $\mathbf{x}_{\lambda}$ for the entry-wise soft thresholding $(\mathbf{x}-\lambda)_{+}$, so that $\hat{X}_{\lambda}(Y)=$ $U_{Y} \cdot\left(\mathbf{y}_{\lambda}\right)_{\Delta} \cdot V_{Y}^{\prime}$ is the SVST denoiser. By invariance of the Frobenius norm to orthogonal transformations, we have

$$
\begin{aligned}
\left\|\hat{X}_{\lambda}\left(Y_{\mu}\right)-\mu X_{0}\right\|_{F}^{2} & =\left\|\mu X_{0}-U_{\mu} \cdot\left(\mathbf{y}_{\mu, \lambda}\right)_{\Delta} \cdot V_{\mu}^{\prime}\right\|_{F}^{2}=\left\|U_{\mu}^{\prime} \cdot \mu X_{0} \cdot V_{\mu}-\left(\mathbf{y}_{\mu, \lambda}\right)_{\Delta}\right\|_{F}^{2} \\
& =\left\|\left(U_{\mu}^{\prime} \cdot \mu X_{0} \cdot V_{\mu}\right)_{\Delta}-\left(\mathbf{y}_{\mu, \lambda}\right)_{\Delta}\right\|_{F}^{2}+\left\|\mu X_{0}\right\|_{F}^{2}-\left\|\left(U_{\mu}^{\prime} \cdot \mu X_{0} \cdot V_{\mu}\right)_{\Delta}\right\|_{F}^{2} .
\end{aligned}
$$

Now, observe that the off diagonal entries of the matrix

$$
\mathbf{y}_{\Delta}=U_{\mu}^{\prime} \cdot \mu X_{0} \cdot V_{\mu}+\frac{1}{\sqrt{n}} U_{\mu}^{\prime} \cdot Z \cdot V_{\mu}
$$

vanish, so that

$$
U_{\mu}^{\prime} \cdot \mu X_{0} \cdot V_{\mu}-\left(U_{\mu}^{\prime} \cdot \mu X_{0} \cdot V_{\mu}\right)_{\Delta}=\frac{1}{\sqrt{n}} U_{\mu}^{\prime} \cdot Z \cdot V_{\mu}-\frac{1}{\sqrt{n}}\left(U_{\mu}^{\prime} \cdot Z \cdot V_{\mu}\right)_{\Delta},
$$

and hence by invariance of the Frobenius norm

$$
\left\|\mu X_{0}\right\|_{F}^{2}-\left\|\left(U_{\mu}^{\prime} \cdot \mu X_{0} \cdot V_{\mu}\right)_{\Delta}\right\|_{F}^{2}=\frac{1}{n}\|Z\|_{F}^{2}-\frac{1}{n}\left\|\left(U_{\mu}^{\prime} \cdot Z \cdot V_{\mu}\right)_{\Delta}\right\|_{F}^{2} .
$$

We thus obtain

$$
\left\|\hat{X}_{\lambda}\left(Y_{\mu}\right)-\mu X_{0}\right\|_{F}^{2}=\left\|\left(U_{\mu}^{\prime} \cdot \mu X_{0} \cdot V_{\mu}\right)_{\Delta}-\left(\mathbf{y}_{\mu, \lambda}\right)_{\Delta}\right\|_{F}^{2}+\frac{1}{n}\|Z\|_{F}^{2}-\frac{1}{n}\left\|\left(U_{\mu}^{\prime} \cdot Z \cdot V_{\mu}\right)_{\Delta}\right\|_{F}^{2} .
$$

Introducing the "pinching" [6] notation

$$
\begin{aligned}
& \mathbf{x}_{\mu}=\mu\left(U_{\mu}^{\prime} \cdot X_{0} \cdot V_{\mu}\right)_{\Delta} \\
& \mathbf{z}_{\mu}=\frac{1}{\sqrt{n}}\left(U_{\mu}^{\prime} \cdot Z \cdot V_{\mu}\right)_{\Delta}
\end{aligned}
$$


so that $\mathbf{y}_{\mu}=\mathbf{x}_{\mu}+\mathbf{z}_{\mu}$, we get

$$
\left\|\hat{X}_{\lambda}\left(Y_{\mu}\right)-\mu X_{0}\right\|_{F}^{2}=\left\|\mathbf{y}_{\mu, \lambda}-\mathbf{x}_{\mu}\right\|_{2}^{2}+\frac{1}{n}\|Z\|_{F}^{2}-\left\|\mathbf{z}_{\mu}\right\|_{2}^{2} .
$$

Therefore,

$$
\begin{aligned}
& \lim _{\mu \rightarrow \infty} R\left(\hat{X}_{\lambda}, \mu X_{0}\right)= \\
= & \lim _{\mu \rightarrow \infty}\left(\frac{1}{m} \mathbb{E}\left\|\mathbf{y}_{\mu, \lambda}-\mathbf{x}_{\mu}\right\|_{2}^{2}+1-\frac{1}{m} \mathbb{E}\left\|\mathbf{z}_{\mu}\right\|_{2}^{2}\right) \\
= & \frac{1}{m} \sum_{i=1}^{r} \lim _{\mu \rightarrow \infty} \mathbb{E}\left(\left(y_{\mu, i}-\lambda\right)_{+}-x_{\mu, i}\right)^{2} \\
+ & \frac{1}{m} \sum_{i=r+1}^{m} \lim _{\mu \rightarrow \infty} \mathbb{E}\left(\left(y_{\mu, i}-\lambda\right)_{+}-x_{\mu, i}\right)^{2} \\
- & \frac{1}{m} \sum_{i=1}^{r} \lim _{\mu \rightarrow \infty} \mathbb{E}\left(z_{\mu, i}\right)^{2} \\
- & \frac{1}{m} \sum_{i=r+1}^{m} \lim _{\mu \rightarrow \infty} \mathbb{E}\left(z_{\mu, i}\right)^{2} \\
+ & 1 .
\end{aligned}
$$

We now proceed to evaluate each of the terms (3.11), (3.12) and (3.13) in turn. Starting with (3.11), by Lemma 15, pointwise $\lim _{\mu \rightarrow \infty} y_{\mu, i}=\infty$, so that there exists $\mu_{0}$ such that $\left(y_{\mu, i}-\lambda\right)_{+}-x_{\mu, i}=y_{\mu, i}-\lambda-x_{\mu, i}=z_{\mu, i}-\lambda$ for all $i$ and $\mu>\mu_{0}$. In particular

$$
\lim _{\mu \rightarrow \infty} \mathbb{E}\left(\left(y_{\mu, i}-\lambda\right)_{+}-x_{\mu, i}\right)^{2}=\lim _{\mu \rightarrow \infty} \mathbb{E}\left(z_{\mu, i}-\lambda\right)^{2}
$$

for $1 \leq i \leq r$, and therefore

$$
\lim _{\mu \rightarrow \infty} \mathbb{E} \sum_{i=1}^{r}\left(\left(y_{\mu, i}-\lambda\right)_{+}-x_{\mu, i}\right)^{2}=\lim _{\mu \rightarrow \infty} \sum_{i=1}^{r} \mathbb{E} z_{\mu, i}^{2}+r \lambda^{2}+2 \lambda \lim _{\mu \rightarrow \infty} \mathbb{E} \sum_{i=1}^{r} z_{\mu, i} .
$$

Since $\sum_{i=1}^{r} z_{\mu, i} \leq \sqrt{r \sum_{i=1}^{r} z_{\mu, i}^{2}} \leq \sqrt{\frac{r}{n}\|Z\|_{F}^{2}}$ and $\mathbb{E}\|Z\|_{F}^{2}=m n$, by dominated convergence and the first part of Lemma 16, $\sum_{i=1}^{r} z_{\mu, i}$ converges in expectation $\mu \rightarrow \infty$ to a zero-mean Gaussian random variable, so that

$$
\lim _{\mu \rightarrow \infty} \mathbb{E} \sum_{i=1}^{r} z_{\mu, i}=0
$$


We conclude that

$$
\lim _{\mu \rightarrow \infty} \mathbb{E} \sum_{i=1}^{r}\left(\left(y_{\mu, i}-\lambda\right)_{+}-x_{\mu, i}\right)^{2}=\lim _{\mu \rightarrow \infty} \sum_{i=1}^{r} \mathbb{E} z_{\mu, i}^{2}+r \lambda^{2} .
$$

Turning to $(3.12)$, let $\left(\zeta_{1}, \ldots, \zeta_{m-r}\right)=\zeta\left(X_{0}, Z \mid \mathbf{X}\right)$ as in Definition 4. By the second part of Lemma 16 we have $\lim _{\mu \rightarrow \infty} x_{\mu, i}=0$ and $\lim _{\mu \rightarrow \infty} z_{\mu, i}=$ $\zeta_{r-i} / \sqrt{n}$ for $r+1 \leq i \leq m$. Again since $\mathbb{E} z_{\mu, i}^{2} \leq \mathbb{E}\|Z\|_{F}^{2}=m n$ for all $\mu, i$, by dominated convergence

$$
\lim _{\mu \rightarrow \infty} \mathbb{E}\left(\left(y_{\mu, i}-\lambda\right)_{+}-x_{\mu, i}\right)^{2}=\frac{1}{n} \mathbb{E}\left(\zeta_{i-r}-\sqrt{n} \lambda\right)_{+}^{2} .
$$

Finally, as for (3.13), by similar reasoning we find that

$$
\lim _{\mu \rightarrow \infty} \mathbb{E}\left(z_{\mu, i}\right)^{2}=\frac{1}{n} \mathbb{E}\left(\zeta_{i-r}\right)^{2}
$$

Collecting the terms in (3.10), we find that the sum does not depend on the particular choice of the signal matrix $X_{0}$, and in fact

$$
\begin{aligned}
R\left(\hat{X}_{\lambda}, \mu X_{0}\right) & =1+\frac{r}{m} \lambda^{2} \\
& +\frac{1}{n m} \sum_{i=1}^{m-r} \mathbb{E}\left(\zeta_{i}-\sqrt{n} \lambda\right)_{+}^{2}+\frac{1}{n m} \sum_{i=1}^{m-r} \mathbb{E} \zeta_{i}^{2}
\end{aligned}
$$

Now,

$$
\frac{1}{n m} \sum_{i=1}^{m-r} \mathbb{E}\left(\zeta_{i}-\sqrt{n} \lambda\right)_{+}^{2}=\alpha \frac{n-r}{m n} \sum_{i=1}^{m-r} \mathbb{E}\left(\frac{\zeta_{i}}{\sqrt{n-r}}-\frac{\lambda}{\sqrt{1-r / n}}\right)_{+}^{2},
$$

with $\alpha=1$ in case Mat and $\alpha=1 / 2$ in case Sym. This factor in case Sym follows from the fact that, in this case, with probability $1 / 2$ we have $z_{i}<0$, and that, conditional on $z_{i}>0, z_{i}$ follows the same distribution as in case Mat. Observe that in the case Mat, $\left\{\zeta_{i} / \sqrt{n-r}\right\}_{i=1 \ldots m-r}$ are the eigenvalues of a standard central Wishart matrix $\sim W_{m-r}(I, n-r)$, so that

$$
\frac{1}{m n} \sum_{i=1}^{m-r} \mathbb{E}\left(\zeta_{i}-\sqrt{n} \lambda\right)_{+}^{2}=\alpha \frac{(n-r)}{m n} \sum_{i=1}^{m-r} w_{i}\left(\frac{\lambda}{\sqrt{1-r / n}} ; m-r ; n-r\right),
$$

where $w_{i}$ was defined in (2.4). 
To complete the proof, we note that $\sum_{i=1}^{m-r} \zeta_{i}^{2}$ is the Frobenius norm of $Z_{(m-r) \times(n-r)}$, hence equals $(m-r)(n-r)$ in expectation, to the effect that

$$
\frac{1}{m n} \mathbb{E} \sum_{i=1}^{m-r} \zeta_{i}^{2}=\frac{(m-r)(n-r)}{m n}=1-\frac{r}{m}-\frac{r}{n}+\frac{r^{2}}{m n} .
$$

Setting $\Lambda=\lambda / \sqrt{1-r / n}$, we collect the terms and recover (2.3) as required.

4. Convexity of the minimax risk. In this supplemental section we prove Lemma 6 and Lemma 9, whereby the finite- $n$ and asymptotic minimax risk are both convex in $\Lambda$.

4.1. Proof of Lemma 6. Differentiating (2.4) under the integral w.r.t $\Lambda$, since the upper integration limit does not depend on $\Lambda$, we get

$$
\begin{aligned}
\frac{d}{d \Lambda} w_{i}(\Lambda ; m, n) & =\frac{d}{d \Lambda} \int_{\Lambda^{2}}^{\infty}(\sqrt{t}-\Lambda)^{2} d W_{i}(m, n)(t) \\
& =-\left(\sqrt{\Lambda^{2}}-\Lambda\right)^{2} \frac{d W_{i}(m, n)}{d t}\left(\Lambda^{2}\right) \cdot(2 \Lambda)+\int_{\Lambda^{2}}^{\infty} \frac{d}{d \Lambda}(\sqrt{t}-\Lambda)^{2} d W_{i}(m, n) \\
& =-2 \int_{\Lambda^{2}}^{\infty}(\sqrt{t}-\Lambda) d W_{i}(m, n)
\end{aligned}
$$

Differentiating w.r.t $\Lambda$ again, the boundary terms vanish again and we get

$$
\frac{d^{2}}{d \Lambda^{2}} w_{i}(\Lambda ; m, n)=-2 \int_{\Lambda^{2}}^{\infty} \frac{d}{d \Lambda}(\sqrt{t}-\Lambda) d W_{i}(m, n)=2 \int_{\Lambda^{2}}^{\infty} d W_{i}(m, n) .
$$

Therefore, by (2.3) we have

$$
\begin{aligned}
\frac{d}{d \Lambda} \mathbf{M}_{n}(\Lambda ; r, m, \alpha) & =\frac{d}{d \Lambda} \frac{r(n-r)}{m n} \Lambda^{2}+\alpha \frac{(n-r)}{m n} \sum_{i=1}^{m-r} \frac{d}{d \Lambda} w_{i}(\Lambda ; m-r ; n-r) \\
& =2 \frac{r(n-r)}{m n} \Lambda-2 \int_{\Lambda^{2}}^{\infty}(\sqrt{t}-\Lambda) d W_{i}(m, n) .
\end{aligned}
$$


and

$$
\begin{aligned}
\frac{d^{2}}{d \Lambda^{2}} \mathbf{M}_{n}(\Lambda ; r, m, \alpha) & =\frac{d^{2}}{d \Lambda^{2}} \frac{r(n-r)}{m n} \Lambda^{2}+\alpha \frac{(n-r)}{m n} \sum_{i=1}^{m-r} \frac{d^{2}}{d \Lambda^{2}} w_{i}(\Lambda ; m-r ; n-r) \\
& =2 \frac{r(n-r)}{m n}+2 \alpha \frac{(n-r)}{m n} \sum_{i=1}^{m-r} \int_{\Lambda^{2}}^{\infty} d W_{i}(m-r, n-r)>0 .
\end{aligned}
$$

Therefore, $\Lambda \mapsto \mathbf{M}_{n}(\Lambda ; r, m, \alpha)$ is convex on $[0, \infty)$ with

$$
\begin{aligned}
\frac{d}{d \Lambda} \mathbf{M}_{n}(0 ; r, m, \alpha) & <0 \quad \text { and } \\
\lim _{\Lambda \rightarrow \infty} \frac{d}{d \Lambda} \mathbf{M}_{n}(\Lambda ; r, m, \alpha) & >0
\end{aligned}
$$

and the lemma follows.

4.2. Proof of Lemma 9. Note that (2.9) is conveniently expressed as

$$
\begin{aligned}
\mathbf{M}(\Lambda ; \rho, \tilde{\rho}, \alpha) & =\rho+\tilde{\rho} \\
& +(1-\tilde{\rho})\left[\rho \Lambda^{2}+\alpha(1-\rho) \int_{\Lambda^{2}}^{\gamma_{+}}(\sqrt{t}-\Lambda)^{2} p_{\gamma}(t) d t\right] .
\end{aligned}
$$

Differentiating the righmost term of (4.1) under the integral, we get

$$
\begin{aligned}
\frac{d}{d \Lambda} \int_{\Lambda^{2}}^{\gamma_{+}}(\sqrt{t}-\Lambda)^{2} p_{\gamma}(t) d t & =\left(\sqrt{\gamma_{+}}-\Lambda\right)^{2} p_{\gamma}\left(\gamma_{+}\right) \cdot \frac{\partial \gamma_{+}}{\partial \Lambda}-\left(\sqrt{\Lambda^{2}}-\Lambda\right)^{2} p_{\gamma}\left(\Lambda^{2}\right) \cdot \\
& +\int_{\Lambda^{2}}^{\gamma_{+}} \frac{\partial}{\partial \Lambda}(\sqrt{t}-\Lambda)^{2} p_{\gamma}(t) d t
\end{aligned}
$$

Since $p_{\gamma}\left(\gamma_{+}\right)=0$, both boundary terms vanish and therefore

$$
\frac{d}{d \Lambda} \int_{\Lambda^{2}}^{\gamma_{+}}(\sqrt{t}-\Lambda)^{2} p_{\gamma}(t) d t=-2 \int_{\Lambda^{2}}^{\gamma_{+}}(\sqrt{t}-\Lambda) p_{\gamma}(t) d t=-2 P_{\gamma}\left(\Lambda^{2} ; \frac{1}{2}\right)+2 \Lambda P_{\gamma}\left(\Lambda^{2} ; 0\right),
$$

where $P_{\gamma}$ was defined in (2.8). Differentiating w.r.t $\Lambda$ again, the boundary terms vanish again and we get

$$
\frac{d^{2}}{d \Lambda^{2}} \int_{\Lambda^{2}}^{\gamma_{+}}(\sqrt{t}-\Lambda)^{2} p_{\gamma}(t) d t=2 \int_{\Lambda^{2}}^{\gamma_{+}} p_{\gamma}(t) d t=2 P_{\gamma}\left(\Lambda^{2} ; 0\right)
$$


By (4.1) we obtain

$$
\begin{aligned}
(4.2) \frac{d}{d \Lambda} \mathbf{M}(\Lambda ; \rho, \tilde{\rho}, \alpha) & =2(1-\tilde{\rho}) \rho \Lambda \\
& +2 \alpha(1-\rho)(1-\tilde{\rho})\left(\Lambda P_{\gamma}\left(\Lambda^{2} ; 0\right)-P_{\gamma}\left(\Lambda^{2} ; \frac{1}{2}\right)\right) .
\end{aligned}
$$

and

$$
\frac{d^{2}}{d \Lambda^{2}} \mathbf{M}(\Lambda ; \rho, \tilde{\rho}, \alpha)=2(1-\tilde{\rho}) \rho+2 \alpha(1-\rho)(1-\tilde{\rho}) P_{\gamma}\left(\Lambda^{2} ; 0\right)>0 .
$$

Therefore, $\Lambda \mapsto \mathbf{M}(\Lambda ; \rho, \tilde{\rho}, \alpha)$ is convex on $\left[0, \gamma_{+}\right]$with

$$
\begin{aligned}
\frac{d}{d \Lambda} \mathbf{M}(0 ; \rho, \tilde{\rho}, \alpha) & <0 \quad \text { and } \\
\frac{d}{d \Lambda} \mathbf{M}\left(\gamma_{+} ; \rho, \tilde{\rho}, \alpha\right) & >0
\end{aligned}
$$

and the lemma follows.

5. Additional main results. In this supplemental section we prove Theorem 5, Theorem 6 and Theorem 7.

5.1. Proof of Theorem 5. When $\beta=1, \gamma_{+}=4$ and $\gamma_{-}=0$ in (2.7). Changing the integration variable by $t \mapsto t^{2}$ in $(2.7)$ we get

$$
P_{1}(x ; k)=\frac{1}{\pi} \int_{x}^{2} t^{2 k} \sqrt{4-t^{2}} d t,
$$

namely the $k$-th incomplete moment of the Quarter Circle law. Substituting this and $\rho=\tilde{\rho}=1$ into (2.9) we recover (2.16). The identities (2.17), (2.18) and (2.19) may be directly verified by differentiation.

To show that $\Lambda_{*}(\rho, \rho, \alpha)$ satisfies (2.21), observe that the condition (7.1), which is equivalent to the general minimizer characterization (2.15), may in the case $\beta=1$ be written in the equivalent form

$$
\frac{1}{\pi} \int_{\Lambda}^{2}(t-\Lambda) \sqrt{4-t^{2}} d t=\frac{\Lambda \rho}{1-\rho} .
$$

Clearly any solution $\Lambda_{*}$ satisfies $0 \leq \Lambda_{*} \leq 2$ and we define

$$
\theta_{\alpha}(\rho)=\arcsin \left(\Lambda_{*}(\rho, \rho, \alpha) / 2\right) .
$$


Changing the integration variable $t \mapsto 2 \sin \psi$ and substituting $\Lambda=2 \sin \theta$ in (5.2), we find that (5.2) is equivalent to

$$
\frac{4}{\pi} \int_{\theta}^{\pi / 2}(\sin \psi-\sin \theta) \cos ^{2} \psi d \psi=\frac{\rho}{1-\rho} \sin \theta,
$$

which is in turn equivalent to (2.21) by elementary integration.

Our result regarding parametric representation of the minimax AMSE curve for $\beta=1$ follows immediately:

5.2. Proof of Theorem \%. The curve is parametrized using the parameter $\theta$ above. In each of the cases $M a t$ and $S y m$, the formula is obtained by solving (2.21) for $\rho$ to obtain $\rho(\theta)$, and simplifying $\mathbf{M}(\Lambda(\theta) ; \rho(\theta), \rho(\theta), \alpha)$, where $\Lambda(\theta)=2 \sin \theta$ and $\mathbf{M}$ is defined in (2.16). We omit the elementary algebra.

Toward the proof of Theorem 6 , we recall a trivial fact about convergence of minimizers.

Lemma 17. Let $f_{n}:[a, b] \rightarrow \mathbb{R}$ be a sequence of continuous functions on $[a, b] \subset \mathbb{R}$ and assume that $\left\{f_{n}\right\}$ converges pointwise to $f:[a, b] \rightarrow \mathbb{R}$. If $x_{n} \in[a, b]$ is the unique minimizer of $f_{n},(n=1,2, \ldots)$, and $x \in[a, b]$ is the unique minimizer of $f$, then $\lim _{n \rightarrow \infty} x_{n}=x$.

Proof. Let $\left\{x_{n_{k}}\right\}$ be a convergent subsequence of $\left\{x_{n}\right\}$, and write

$$
\lim _{k \rightarrow \infty} x_{n_{k}}=y
$$

It is enough to show that $y=x$. Since $f_{n}$ is continuous on a compact interval, it is uniformly continuous, hence $f_{n_{k}}\left(x_{n_{k}}\right) \rightarrow f(y)$. Since $x_{n_{k}}$ is a minimizer of $f_{n_{k}}$, for all $k$ we have $f_{n_{k}}\left(x_{n_{k}}\right) \leq f_{n_{k}}(x)$. In the limit $k \rightarrow \infty$ this inequality yields $f(y) \leq f(x)$. Since $x$ is a minimizer of $f, f(x) \leq f(y)$. Therefore $f(x)=f(y)$. It follows that $y$ is a minimizer of $f$, which is unique by assumption, so that $x=y$.

\subsection{Proof of Theorem 6. Define}

$$
\Lambda_{*}^{n}(r, m, \alpha)=\operatorname{argmin}_{\Lambda} \mathbf{M}_{n}(\Lambda ; r, m, \alpha),
$$

and recall the definition of $\Lambda_{*}(\rho, \beta, \alpha)$ in (2.12). By Theorem 1, Lemma 5 and Lemma 8 , the function sequence $\Lambda \mapsto \mathbf{M}_{n}(\Lambda ; r, m, \alpha)$ converge pointwise 
to the function $\Lambda \mapsto \mathbf{M}(\Lambda, \rho, \tilde{\rho}, \alpha)$ on $\Lambda \in\left[0, \gamma_{+}\right]$. We invoke Lemma 17 to obtain that the minimizers of the former converge to the minimizer of the latter, namely

$$
\lim _{n \rightarrow \infty} \Lambda_{*}^{n}(r, m, \alpha)=\Lambda_{*}(\rho, \beta, \alpha)
$$

Observe that

$$
\begin{aligned}
& \lambda_{*}(m, n, r \mid \mathbf{X})=\operatorname{argmin}_{\lambda} \sup _{\substack{X_{0} \in \mathbf{X}_{m, n} \\
\operatorname{rank}\left(X_{0}\right) \leq r}} \frac{1}{m n} \mathbb{E}_{X_{0}}\left\|\hat{X}_{\lambda}\left(X_{0}+Z\right)-X_{0}\right\|_{F}^{2} \\
& =\sqrt{n} \cdot \operatorname{argmin}_{\lambda} \sup _{\substack{X_{0} \in \mathbf{X}_{m, n} \\
\operatorname{rank}\left(X_{0}\right) \leq r}} \frac{1}{m n} \mathbb{E}_{X_{0}}\left\|\hat{X}_{\lambda}\left(X_{0}+Z / \sqrt{n}\right)-X_{0}\right\|_{F}^{2} \\
& =\sqrt{n} \cdot \operatorname{argmin}_{\lambda} \mathbf{M}_{n}\left(\frac{\lambda}{\sqrt{1-r / n}} ; r, m, \alpha\right) \\
& =\sqrt{n} \cdot \sqrt{1-r / n} \cdot \operatorname{argmin}_{\Lambda} \mathbf{M}_{n}(\Lambda ; r, m, \alpha) \\
& =\sqrt{n} \cdot \sqrt{1-r / n} \cdot \Lambda_{*}^{n}(r, m, \alpha) \text {. }
\end{aligned}
$$

with $\alpha=1$ for case Mat and $\alpha=1 / 2$ for case Sym. Since $\lim _{n \rightarrow \infty} \sqrt{1-r / n}=$ $\sqrt{1-\beta \rho}$, we thus have

$\lim _{n \rightarrow \infty} \frac{1}{\sqrt{n}} \lambda_{*}(m, n, r \mid \mathbf{X})=\lim _{n \rightarrow \infty} \sqrt{1-r / n} \cdot \Lambda_{*}^{n}(r, m, \alpha)=\sqrt{1-\beta \rho} \cdot \Lambda_{*}(\rho, \beta, \alpha)$,

where we have used (5.6) .

We proceed to evaluate the $\operatorname{limit}_{\lim _{\rho \rightarrow 0}} \mathcal{M}(\rho, \beta \mid \mathbf{X}) / \rho$.

5.4. Proof of Theorem 8. We first show that

$$
\lim _{\rho \rightarrow 0} \frac{1}{\rho} \int_{\Lambda_{*}^{2}(\rho)}^{\gamma_{+}(\rho)}\left(\sqrt{t}-\Lambda_{*}(\rho)\right)^{2} p_{\gamma(\rho)}(t) d t=0 .
$$

Observe that by (2.15), which is equivalent to (7.1), the minimizer $\Lambda_{*}$ satisfies

$$
\int_{\Lambda_{*}^{2}(\rho)}^{\gamma_{+}(\rho)}\left[\sqrt{t}-\Lambda_{*}(\rho)\right] p_{\gamma(\rho)}(t) d t=\frac{\rho \Lambda_{*}(\rho)}{\alpha(1-\rho)}
$$


so that

$$
\lim _{\rho \rightarrow 0} \int_{\Lambda_{*}^{2}(\rho)}^{\gamma_{+}(\rho)}\left[\sqrt{t}-\Lambda_{*}(\rho)\right] p_{\gamma(\rho)}(t) d t=0 .
$$

Differentiating (5.7) under the integral sign, it remains to show that

$$
\lim _{\rho \rightarrow 0} \int_{\Lambda_{*}^{2}(\rho)}^{\gamma_{+}(\rho)} t^{k / 2} \frac{\partial}{\partial \rho} p_{\gamma(\rho)}(t) d t=0
$$

for $k=0,1,2$. Note that $\lim _{\rho \rightarrow 0} \Lambda_{*}^{2}(\rho)=\lim _{\rho \rightarrow 0} \gamma_{+}(\rho)=(1+\sqrt{\beta})^{2}$. However, it is easy to verify that $\partial p_{\gamma(\rho)} / \partial \rho \leq C \cdot\left(\gamma_{+}(\rho)-t\right)^{-1 / 2}$ in a neighborhood of $(1+\sqrt{\beta})^{2}$, for an appropriate constant $C$, so that

$$
\lim _{\rho \rightarrow 0} \int_{\Lambda_{*}^{2}(\rho)}^{\gamma_{+}(\rho)} t^{k / 2} \frac{\partial}{\partial \rho} p_{\gamma(\rho)}(t) d t \leq\left.\lim _{\rho \rightarrow 0} C \sqrt{\gamma_{+}(\rho)-t}\right|_{\Lambda_{*}^{2}(\rho)} ^{\gamma_{+}(\rho)}=0 .
$$

We now proceed to calculate the required limit. In the case $M a t$, by (2.13) we have

$$
\lim _{\rho \rightarrow 0} \Lambda_{*}(\rho, \beta, \alpha)=1+\sqrt{\beta}
$$

Therefore

$$
\begin{aligned}
\lim _{\rho \rightarrow 0} \frac{1}{\rho} \mathcal{M}(\rho, \beta \mid \text { Mat }) & =\lim _{\rho \rightarrow 0} \frac{1}{\rho} \mathbf{M}\left(\Lambda_{*}(\rho, \beta, 1), \rho, \tilde{\rho}, 1\right) \\
& =\lim _{\rho \rightarrow 0} \frac{1}{\rho}\left(\rho+\tilde{\rho}+\rho \tilde{\rho}+\rho(1-\tilde{\rho}) \Lambda_{*}^{2}(\rho, \beta, 1)\right) \\
& =\lim _{\rho \rightarrow 0}\left(1+\beta+\rho \beta+(1-\rho \beta)(1+\sqrt{\beta})^{2}\right) \\
& =1+\beta+(1+\sqrt{\beta})^{2}=2(1+\sqrt{\beta}+\beta),
\end{aligned}
$$

where we have used (5.7) and the relation $\tilde{\rho}=\beta \rho$. The calculation for case Sym is identical.

\section{Singular-value denoisers can only improve in worst-case.}

6.1. Proof of Lemma 10. Let $O_{n}$ denote the orthogonal group in $M_{n \times n}$ and let $O_{m} \times O_{n}$ act on $M_{m \times n}$ by $(U, V): X \mapsto U \cdot X \cdot V^{\prime}$. Recall that a decision rule $\hat{X}$ satisfying $U \hat{X}(Y) V^{\prime}=\hat{X}\left(U Y V^{\prime}\right)$ for all $(U, V) \in G$ is called 
equivariant with respect this this group action (see [19, def. 2.5]) In [30, cor. 7 ] it is shown that $\mathcal{D}$ coincides with the family of equivariant decision rules (see also [21, prop. 5.1]).

Now, let $\hat{X}_{1}: M_{m \times n} \rightarrow M_{m \times n}$ be an arbitrary measurable matrix denoiser. We now invoke the generalized Hunt-Stein Theorem for estimation $[17,3]$, which in our situation ${ }^{2}$ states that for the chosen denoiser $\hat{X}_{1}: M_{m \times n} \rightarrow M_{m \times n}$ there exists an equivariant denoiser $\hat{X} \in \mathcal{D}$ such that

$$
\text { (6.1) } \sup _{(U, V) \in O_{m} \times O_{n}} R\left(\hat{X}, U \cdot X_{0} \cdot V^{\prime}\right) \leq \sup _{(U, V) \in O_{m} \times O_{n}} R\left(\hat{X}_{1}, U \cdot X_{0} \cdot V^{\prime}\right),
$$

for all $X_{0} \in M_{m \times n}$ with $\operatorname{rank}\left(X_{0}\right) \leq r$. Since $\hat{X} \in \mathcal{D}$, we have $R\left(\hat{X}, U \cdot X_{0}\right.$. $\left.V^{\prime}\right)=R\left(\hat{X}, X_{0}\right)$ for all $(U, V) \in O_{m} \times O_{n}$. Taking the supremum on both sides of (6.1), we obtain

$$
\sup _{\substack{X_{0} \in M_{m \times n} \\ \operatorname{rank}\left(X_{0}\right) \leq r}} R\left(\hat{X}, X_{0}\right) \leq \sup _{\substack{X_{0} \in M_{m \times n} \\ \operatorname{rank}\left(X_{0}\right) \leq r}} R\left(\hat{X}_{1}, X_{0}\right),
$$

as desired.

\section{Additional Discussion.}

7.1. Another Connection between natrix SVST and sparse vector estimation: Block Thresholding. The parallels between the problems do not seem accidental. An interesting denoising problem intermediate between sparse vector and low-rank matrices involves block-sparse vectors [12]. Expressing that problem in this paper's notation, the object $X_{0}$ is an $m$-by- $n$ array, and we call the columns "blocks"; so each block has $m$ entries. We assume at most a fraction $\epsilon$ of blocks are nonzero: $\epsilon \cdot n \geq \#\left\{j:\|X(\cdot, j)\|_{2} \neq 0\right\}$. We observe noisy matrix data $Y=X_{0}+Z$ and we consider the block-shrinkage problem:

$$
\begin{aligned}
\left(P_{2,1}\right) \quad \hat{X}_{\lambda} & =\operatorname{argmin}_{X \in M_{m \times n}} \frac{1}{2}\|Y-X\|_{F}^{2} \\
& +\lambda \sum_{j}\|X(\cdot, j)\|_{2} .
\end{aligned}
$$

By the inequalities

$$
\|X\|_{*} \leq \sum_{j}\|X(\cdot, j)\|_{2} \leq \sum_{i j}|X(i, j)|,
$$

\footnotetext{
${ }^{2}$ For the generalized Hunt-Stein Theorem to hold, it is sufficient to show that the group is amenable. Indeed, $O_{m} \times O_{n}$ is compact in the product topology, hence amenable.
} 
(NNP) is a relaxation of $\left(P_{2,1}\right)$, while $\left(P_{2,1}\right)$ is a relaxation of $\left(P_{1}\right)$ applied to $\operatorname{vec}(Y)$.

In the case $m=1$ we recover $\left(P_{1}\right)$ and the soft thresholding procedure. In the case $m>1$ we obtain block thresholding; it promotes reconstructions $\hat{X}_{\lambda}$ where many blocks are fully zero and a small fraction are nonzero; the nonzero blocks are those where $\|X(\cdot, j)\|_{2}>\lambda$.

The chain of inequalities $(7.2)$ places $\left(P_{2,1}\right)$ intermediate between $\left(P_{1}\right)$ and (NNP). All the parallels mentioned so far between soft thresholding and SVST also hold between block soft thresholding and the other two methods.

- All three involve soft thresholding of relevant objects - scalars, column norms, or singular values.

- All three have a least favorable estimand with its nonzero piece "at $\infty "$

- All three have a minimax penalty factor $\lambda^{*}(\varepsilon)$ monotone decreasing in $\varepsilon$.

Moreover, the two parallels of the last section carry over as well. For block thresholding, the minimax MSE involves incomplete moments, this time of the classical $\chi_{m}^{2}$ distribution. The monotonicity of SURE carries through and implies the structure of the least-favorable estimand.

7.2. Block Thresholding and the Minimaxity Gap. We bring up block soft thresholding because of its relevance to the minimaxity gap of SVST that we conjectured in the introduction. Donoho, Johnstone and Montanari [12] considered the following limiting case, where we consider $n \rightarrow \infty$ first, and later $m \rightarrow \infty$. In that setting, the minimax MSE among all measurable procedures under $\varepsilon$-block sparsity, namely,

$$
M_{\text {block }}^{*}(\varepsilon)=\lim _{m \rightarrow \infty} \lim _{n \rightarrow \infty} \inf _{\hat{X}} \sup _{\#\left\{j: X_{0}(\cdot, j) \neq 0\right\} \leq \varepsilon n} \frac{1}{n m} \mathbb{E}\left\|\hat{X}-X_{0}\right\|_{F}^{2},
$$

can be evaluated. It obeys $M_{\text {block }}^{*}(\varepsilon)=\varepsilon$, and they show it can be attained asymptotically by a particularly lovely method: simply apply the JamesStein shrinkage estimator blockwise! On the other hand, the minimax MSE for soft block thresholding can be evaluated:

$$
M_{\text {block }}(\varepsilon)=\lim _{m \rightarrow \infty} \lim _{n \rightarrow \infty} \inf _{\lambda} \sup _{\#\left\{j: X_{0}(\cdot, j) \neq 0\right\} \leq \varepsilon n} \frac{1}{n m} \mathbb{E}\left\|\hat{X}_{\lambda}-X_{0}\right\|_{F}^{2} .
$$

They obtain $M_{\text {block }}(\varepsilon)=2 \varepsilon-\varepsilon^{2}$. Consequently, block soft thresholding is never worse than a factor of 2 from minimaxity, at any level of block sparsity. This bound is achieved as $\varepsilon \rightarrow 0$ :

$$
\lim _{\varepsilon \rightarrow 0} \frac{M_{b l o c k}^{*}(\varepsilon)}{M_{b l o c k}(\varepsilon)}=2
$$


namely, in the high-dimensional limit, under extreme sparsity, block soft thresholding is a factor 2 worse than minimax. These completed results about the minimaxity gap in high-dimensional block soft thresholding are suggestive from the viewpoint of Singular Value Soft Thresholding. Could there be an estimator improving on SVST, and particularly lovely in form?

Acknowledgements. We thank Iain Johnstone, Andrea Montanari and Art Owen for advice at several crucial points, and the anonymous referees for many helpful suggestions. This work was partially supported by NSF DMS 0906812 (ARRA). MG was partially supported by a William R. and Sara Hart Kimball Stanford Graduate Fellowship. and a Technion EE Sohnis Promising Scientist Award.

\section{References.}

[1] Anderson, T. W. (1955). The Integral of a Symmetric Unimodal Function over a Symmetric Convex Set and Some Probability Inequalities. Proceedings of the American Mathematical Society 6 170-176.

[2] Aussel, D. (1998). Subdifferential Properties of Quasiconvex and Pseudoconvex Functions : Unified Approach. Journal of optimization theory and applications 97 29-45.

[3] Bondar, J. V. and Milnes, P. (1981). Amenability: A survey for statistical applications of Hunt-Stein and related conditions on groups. Zeitschrift fr Wahrscheinlichkeitstheorie und verwandte Gebiete 57 103-128.

[4] Candès, E. J., Sing-Long, C. A. and Trzasko, J. D. (2012). Unbiased risk estimates for singular value thresholding and spectral estimators. IEEE Transactions on Signal Processing 61 4643-4657.

[5] Das Gupta, S., Anderson, T. W. and Mudholkar, G. S. (1964). Monotonicity of the power functions of some tests of the multivariate linear hypothesis. The Annals of Mathematical Statistics 35 200-205.

[6] Davis, C. (1963). The Rotation of Eigenvectors by a Perturbation. Journal of Mathematical Analysis and Applications 6 159-173.

[7] Donoho, D. L. and Gavish, M. (2013). Companion website for the article The Phase Transition of Matrix Recovery from Gaussian Measurements Matches the Minimax MSE of Matrix Denoising. http://www . runmycode.org/CompanionSite/Site265. accessed 15 February 2013.

[8] Donoho, D. L. and Gavish, M. (2013). Supplement to "Minimax Risk of Matrix Denoising by Singular Value Thresholding".

[9] Donoho, D. L., Gavish, M. and Montanari, A. (2013). The Phase Transition of Matrix Recovery from Gaussian Measurements Matches the Minimax MSE of Matrix Denoising. Proceedings of the National Academy of Sciences 110 8405-8410.

[10] Donoho, D. L. and Johnstone, I. M. (1994). Minimax risk overl $\ell_{p^{-}}$balls forl $\ell_{q^{-}}$ error. Probability Theory and Related Fields 303 277-303.

[11] Donoho, D. L., Johnstone, I. M., Hoch, J. C. and Stern, A. S. (1992). Maximum entropy and the nearly black object. Journal of the Royal Statistical Society. Series B 54 41-81. 
[12] Donoho, D. L., Johnstone, I. M. and Montanari, A. (2011). Accurate Prediction of Phase Transitions in Compressed Sensing via a Connection to Minimax Denoising. IEEE Transactions on Information Theory 59 3396-3433.

[13] Donoho, D. L. and Gavish, M. (2013). The optimal hard threshold for singular values is $4 / \sqrt{3}$. Stanford University Department of Statistics technical report no. 201304. http://arxiv.org/abs/1305.5870

[14] Rohde, A. and Tsybakov, A. B. (2011). Estimation of high-dimensional low-rank matrices.. Annals of Statistics 39 887-930.

[15] Golub, G. H. and Van Loan, C. F. (1996). Matrix Computations. Johns Hopkins University Press.

[16] Grant, M. and Boyd, S. P. (2010). CVX: Matlab Software for Disciplined Convex Programming.

[17] Kiefer, J. (1957). Invariance, Minimax Sequencial Estimation, and Continuous Time Processes. The Annals of Mathematical Statistics.

[18] Koltchinskis, V., Lounici, K. and Tsybakov, A. B. (2011). Nuclear-norm penalization and optimal rates for noisy low-rank matrix completion. Annals of Statistics 39 2302-2329.

[19] Lehmann, E. L. and Casella, G. (1998). Theory of point estimation, 2nd edition.

[20] Lewis, A. S. (1995). The Convex Analysis of Unitarily Invariant Matrix Functions. Journal of Convex Analysis 2 173-183.

[21] Lewis, A. S. and Sendov, H. S. (2005). Nonsmooth Analysis of Singular Values. Part I: Theory. Set-Valued Analysis 13 213-241.

[22] Lewis, A. S. and Sendov, H. S. (2005). Nonsmooth Analysis of Singular Values. Part II: Applications. Set-Valued Analysis 13 243-264.

[23] Marcenko, V. and Pastur, L. (1967). Distribution of eigenvalues for some sets of random matrices. Mathematics USSR Sbornik 1 457-483.

[24] Oymak, S. and Hassibi, B. (2010). New Null Space Results and Recovery Thresholds for Matrix Rank Minimization.

[25] Oymak, S. and Hassibi, B. (2012). On a Relation between the Minimax Denoising and the Phase Transitions of Convex Functions.

[26] Papadopoulo, T. and Lourakis, M. I. A. (2000). Estimating the jacobian of the singular value decomposition: Theory and applications. In Lecture Notes in Computer Science Vol. 1842/2000 554-570.

[27] Recht, B., Fazel, M. and Parrilo, P. A. (2010). Guaranteed minimum-rank solutions of linear matrix equations via nuclear norm minimization. SIAM review $\mathbf{5 2}$ 471-501.

[28] Recht, B., Xu, W. and Hassibi, B. (2008). Necessary and sufficient conditions for success of the nuclear norm heuristic for rank minimization. In Proceedings of the 47th IEEE conference on Decision and Control Cancun, Mexico.

[29] Recht, B., Xu, W. and Hassibi, B. (2010). Null space conditions and thresholds for rank minimization. Mathematical Programming 127 175-202.

[30] Shabalin, A. and Nobel, A. (2010). Reconstruction of a Low-rank Matrix in the Presence of Gaussian Noise. arXiv preprint arXiv:100\%.4148 http://arxiv.org/ abs $/ 1007.4148$

[31] Stein, C. M. (1981). Estimation of the Mean of a Multivariate Normal Distribution. The annals of Statistics 9 1135-1151.

[32] TANnER, J. and WEI, K. (2012). Normalized iterative hard thresholding for matrix completion.

[33] Zanella, A., Chiani, M. and Win, M. Z. (2009). On the marginal distribution 
of the eigenvalues of Wishart matrices. IEEE Transactions on Communications $\mathbf{5 7}$ 1050-1060.

\author{
Department of Statistics \\ SEquoia Hall \\ 390 Serra Mall \\ StANFORd UNIVERSITY \\ StANFORD, CA 94305-4065 \\ E-MAIL: donoho@stanford.edu \\ gavish@stanford.edu
}

